\title{
A Modular Synthetic Approach to Isosteric Sulfonic Acid Analogues of the Anticoagulant Pentasaccharide Idraparinux
}

\author{
Erika Mezó, Dániel Eszenyi, Eszter Varga, Mihály Herczeg and Anikó Borbás *
}

Department of Pharmaceutical Chemistry, University of Debrecen, Egyetem tér 1, H-4032 Debrecen, Hungary; mezzo.erika@science.unideb.hu (E.M.); eszenyi.daniel@science.unideb.hu (D.E.); esztervargaa@gmail.com (E.V.); herczeg.mihaly@science.unideb.hu (M.H.)

* Correspondence: borbas.aniko@pharm.unideb.hu; Tel.: +36-52-512-900

Academic Editor: Vito Ferro

Received: 7 September 2016; Accepted: 3 November 2016; Published: 11 November 2016

\begin{abstract}
Heparin-based anticoagulants are drugs of choice in the therapy and prophylaxis of thromboembolic diseases. Idraparinux is a synthetic anticoagulant pentasaccharide based on the heparin antithrombin-binding domain. In the frame of our ongoing research aimed at the synthesis of sulfonic acid-containing heparinoid anticoagulants, we elaborated a modular pathway to obtain a series of idraparinux-analogue pentasaccharides bearing one or two primary sulfonic acid moieties. Five protected pentasaccharides with different $C$-sulfonation patterns were prepared by two subsequent glycosylation reactions, respectively, using two monosaccharide and four disaccharide building blocks. Transformation of the protected derivatives into the fully $O$-sulfated, $O$-methylated sulfonic acid end-products was also studied.
\end{abstract}

Keywords: heparin; carbohydrates; glycosylation; sulfonic acid; uronic acid

\section{Introduction}

Venous and arterial thromboembolic disorders, including pulmonary embolism and deep vein thrombosis represent a serious medical and socioeconomic problem worldwide. Untreated thromboembolism leads to cardiac or cerebral infarction or, in more severe cases, to death. Anticoagulants are used in the prevention and treatment of venous thrombosis and in the prevention of systemic embolism [1-4]. The sulfated polysaccharide heparin and its fractionated derivatives have successfully been used in anticoagulant therapy and thromboprophylaxis since the late 1930s until today. Heparin derivatives indirectly inhibit the coagulation enzymes thrombin or factor Xa through activation of the serine protease inhibitor antithrombin, which is an endogenous regulatory protein in the coagulation cascade [5]. Despite their effectiveness in therapy, heparin polysaccharides may incur side effects including inflammation, bleeding or heparin induced thrombocytopenia (HIT) due to its highly polyanionic and heterogeneous nature [6].

After the antithrombin-binding pentasaccharide domain of heparin (1), termed DEFGH, was identified, its closely related synthetic analogue, fondaparinux 2 , has been developed into a novel antithrombotic under the name Arixtra [7,8]. This pentasaccharide selectively inhibits factor Xa and minimizes the bleeding risk and many other unfavorable factors in anticoagulant therapy. Further research efforts led to the development of the non-glycosaminoglycan derivative, idraparinux 3 [9], possessing a simplified structure and an increased anticoagulant activity compared to Arixtra.

The interaction between heparin and antithrombin are primarily mediated by negatively charged groups of heparin and the positively charged lysine and arginine residues from the protein. Structure-activity relationship (SAR) studies of synthetic analogues of heparin pentasaccharides 
revealed that the type of negative charge is crucial; the carboxylate groups cannot be exchanged for sulfate esters, and sulfate moieties cannot be exchanged for phosphate groups without destroying the anticoagulant activity $[1,3]$.

With the aim of developing a novel class of heparinoid anticoagulants, our group has started a programme to study whether the sulfate ester groups of the active pentasaccharide can be exchanged with sulfonic acid moieties without detriment to the antithrombin-binding ability [10-19]. Two pentasaccharide sulfonic acids (4 and 5 ) and the reference compound idraparinux 3 have been prepared until now. An in vitro coagulation study of $\mathbf{2}-\mathbf{5}$ clearly demonstrated that the position and/or number of the sulfonic acid moieties have a substantial impact on the anticoagulant activity (Figure 1). While the disulfonate analogue 4 displayed higher activity than the reference compounds 2 and $\mathbf{3}$, the introduction of a third sulfonic-acid moiety to the terminal sugar unit of compound $\mathbf{5}$ resulted in a dramatic decrease in anti-Xa activity [14]. The difference in the biological activity of the disulfonic and trisulfonic acids was attributed to the different conformation of their L-iduronic-acid residues.

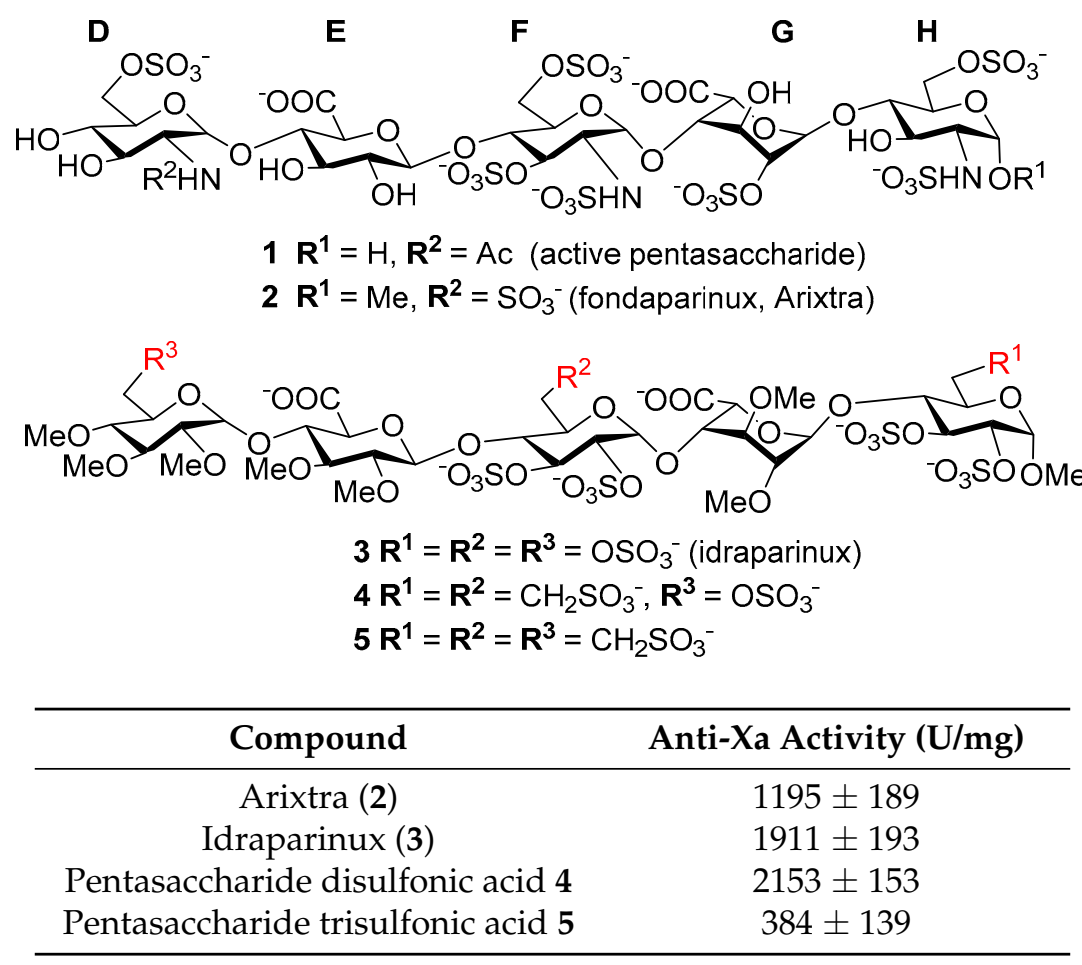

Figure 1. The antithrombin-binding pentasaccharide domain of heparin (1) and structures and factor Xa inhibitory activities of the synthetic analogues 2-5.

These results prompted us to prepare a series of heparinoid pentasaccharides by systematic replacement of the sulfate esters with a sodium sulfonatomethyl moiety for further structure-activity relationship studies. To get an easy access to all possible sulfonic acid isosters of idraparinux bearing the sulfonic acid moieties at primary positions, we elaborated a modular synthetic pathway based on the retrosynthetic analysis of the targeted pentasaccharides (Figure 2). According to this modular approach, the synthesis of all planned pentasaccharide sulfonic acids could be accomplished by using two DE disaccharide donors, two F building blocks and two GH disaccharide acceptors. Multigram-scale syntheses of the 6-deoxy-6-sulfonatomethyl-containing F, DE and GH building blocks have been published recently [17]. Herein, we present the synthesis of the FGH acceptors and assembly of five protected pentasaccharide sulfonic acids via [2+3] block syntheses. Our study to convert the protected mono- and disulfonic acid derivatives into the corresponding fully $O$-sulfated and $O$-methylated end-products via two different reaction sequences is also described. 

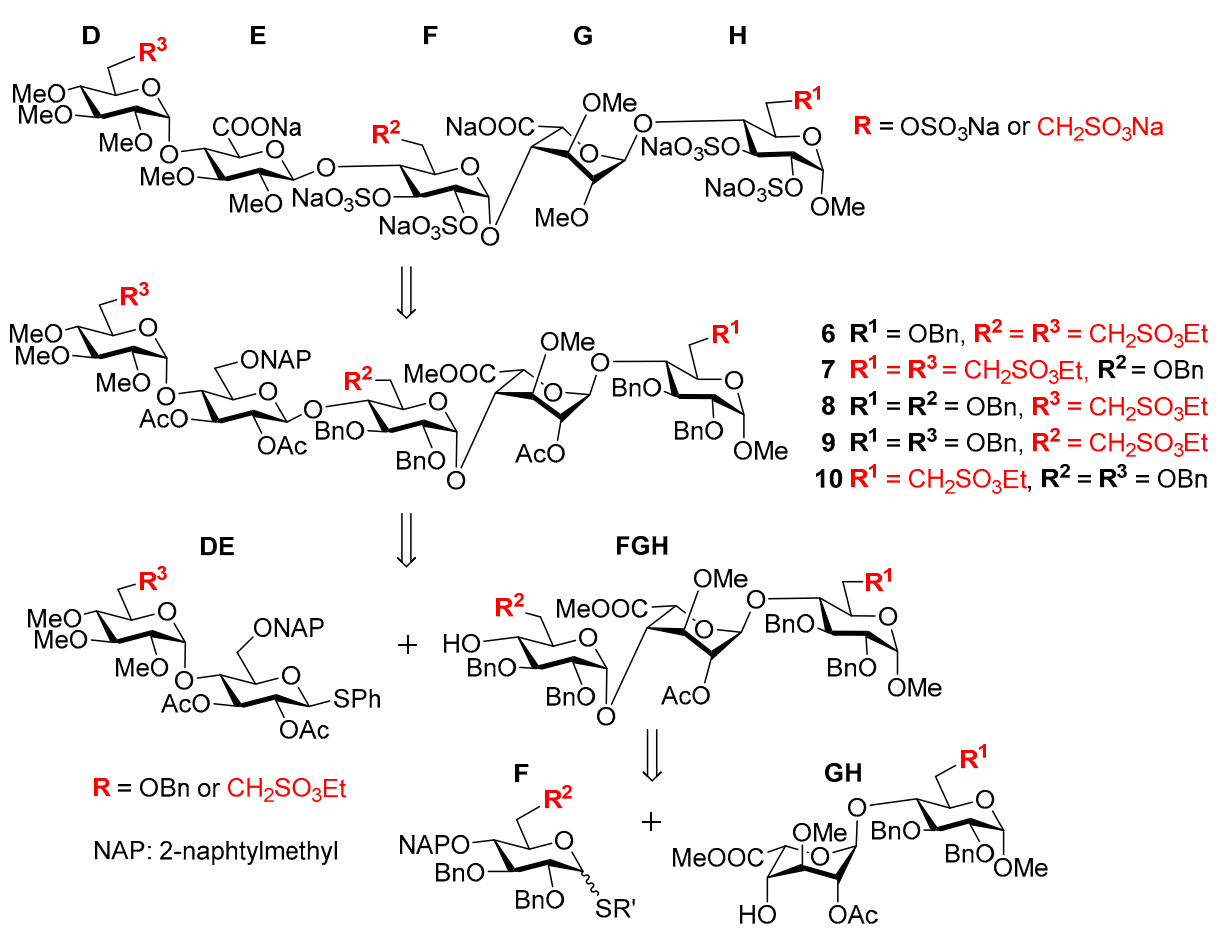

Figure 2. Retrosynthetic analysis for modular [2+3] block syntheses of the targeted pentasaccharide sulfonic acids $\mathbf{6} \mathbf{- 1 0 .}$

\section{Results and Discussion}

\subsection{Synthesis of the Protected Pentasaccharides}

The synthetic route to the FGH trisaccharide acceptors $\mathbf{2 0 - 2 2}$ is shown in Scheme 1. First, the L-idose-containing diol $\mathbf{1 1}$ [18] was converted to iduronide acceptor $\mathbf{1 2}$ by selective oxidation using (2,2,6,6-tetramethylpiperidin-1-yl)oxyl (TEMPO) as the oxidant and [bis(acetoxy)iodo]benzene (BAIB) as the co-oxidant [20,21]. The corresponding sulfonic acid isoster 14 [17] was prepared from 13 [17] in an analogous way. Next, GH disaccharide acceptors $\mathbf{1 2}$ and $\mathbf{1 4}$ were reacted with F monosaccharide donors 15 [16] and 16 [18], respectively, in the presence of $N$-iodosuccinimide (NIS) and trifluoromethanesulfonic acid ( $\mathrm{TfOH})$. This promoter system proved to be highly efficient for stereoselective condensation of the phenylthio-glucoside donor $\mathbf{1 6}$ with either of the acceptors, and the corresponding trisaccharides 18 and 19, with the required $\alpha$-interglycosidic linkage, were obtained in $98 \%$ and $80 \%$ yields, respectively. NIS-TfOH-promoted glycosylation of $\mathbf{1 2}$ with the sulfonatomethyl donor $\mathbf{1 5}$ also occurred with full $\alpha$-selectivity affording trisaccharide $\mathbf{1 7}$ as the only product. However, the yield was moderate due to insufficient conversion of the acceptor. Fortunately, by changing the promotors to NIS-AgOTf, the conversion could significantly be increased, and the yield of $\mathbf{1 7}$ reached $73 \%$. Liberation of the $4-\mathrm{OH}$ group of the terminal glucose unit of the fully protected trisaccharides 17-19 was accomplished by oxidative removal of the (2-naphthyl)methyl (NAP) group using 2,3-dichloro-5,6-dicyano-1,4-benzoquinone (DDQ) as the reagent [22,23], furnishing the FGH acceptors 20-22 in good to excellent yields.

To avoid inefficient glycosylations with glucuronic acid donors of inherent low reactivity, which were observed in earlier syntheses [9,15,24], donors 23 [17] and 24 [13] containing a non-oxidized precursor of the glucuronide unit were used for [2+3] block syntheses of the targeted pentasaccharides (Scheme 2). Condensation reactions of the trisaccharide acceptors 20-22 with the disaccharide donors 23 and 24 were carried out upon NIS-AgOTf or NIS-TfOH activation, respectively. All reactions took place in a stereoselective way providing the protected pentasaccharides $\mathbf{6 - 1 0}$, with the required $\beta$-linkage between units $\mathbf{E}$ and $\mathbf{F}$, in good yields. 


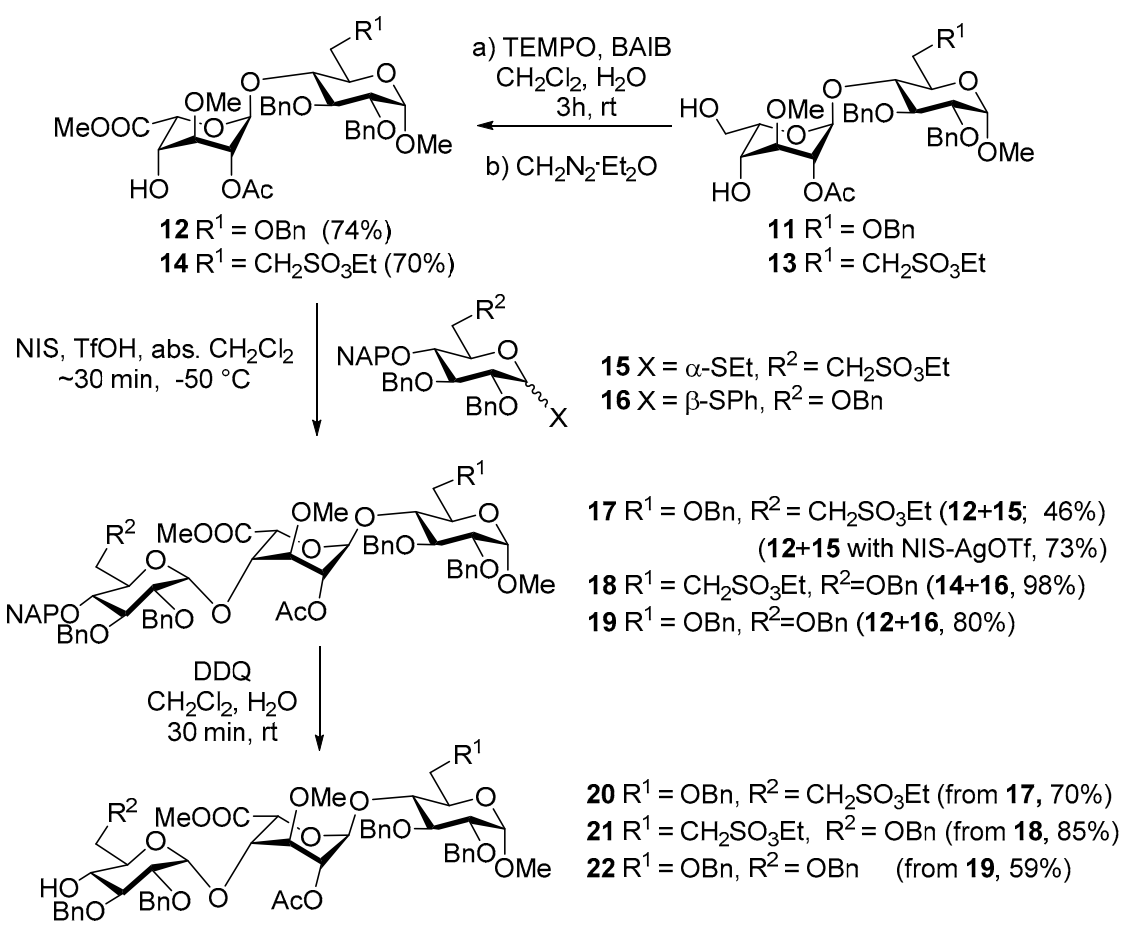

Scheme 1. Preparation of the FGH building blocks.

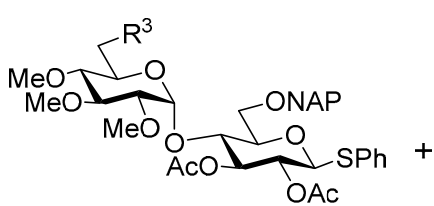

$23 \mathrm{R}^{3}=\mathrm{CH}_{2} \mathrm{SO}_{3} \mathrm{Et}$

$24 \mathrm{R}^{3}=\mathrm{OBn}$

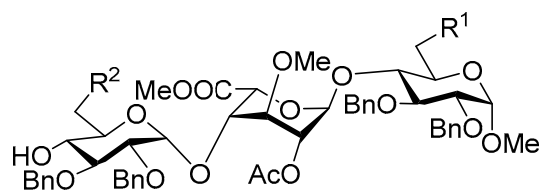

$20 \mathrm{R}^{1}=\mathrm{OBn}, \mathrm{R}^{2}=\mathrm{CH}_{2} \mathrm{SO}_{3} \mathrm{Et}$

$21 \mathrm{R}^{1}=\mathrm{CH}_{2} \mathrm{SO}_{3} \mathrm{Et}, \mathrm{R}^{2}=\mathrm{OBn}$

$22 \mathrm{R}^{1}=\mathrm{OBn}, \mathrm{R}^{2}=\mathrm{OBn}$

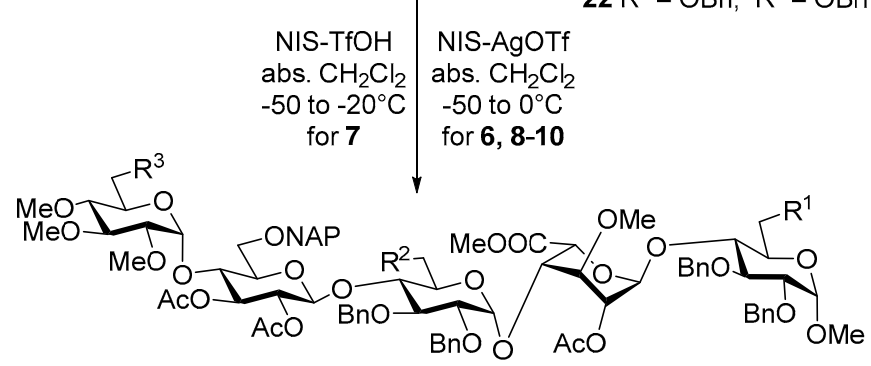

$6 \mathrm{R}^{1}=\mathrm{OBn}, \mathrm{R}^{2}=\mathrm{CH}_{2} \mathrm{SO}_{3} \mathrm{Et}, \mathrm{R}^{3}=\mathrm{CH}_{2} \mathrm{SO}_{3} \mathrm{Et}(20+23,62 \%)$

$7 \mathrm{R}^{1}=\mathrm{CH}_{2} \mathrm{SO}_{3} \mathrm{Et}, \mathrm{R}^{2}=\mathrm{OBn}, \mathrm{R}^{3}=\mathrm{CH}_{2} \mathrm{SO}_{3} \mathrm{Et}(\mathbf{2 1}+\mathbf{2 3}, 63 \%)$

$8 \mathrm{R}^{1}=\mathrm{OBn}, \mathrm{R}^{2}=\mathrm{OBn}, \mathrm{R}^{3}=\mathrm{CH}_{2} \mathrm{SO}_{3} \mathrm{Et} \quad(22+\mathbf{2 3}, 68 \%)$

$9 \mathrm{R}^{1}=\mathrm{OBn}, \mathrm{R}^{2}=\mathrm{CH}_{2} \mathrm{SO}_{3} \mathrm{Et}, \mathrm{R}^{3}=\mathrm{OBn} \quad(20+\mathbf{2 4}, 63 \%)$

$10 \mathrm{R}^{1}=\mathrm{CH}_{2} \mathrm{SO}_{3} \mathrm{Et}, \mathrm{R}^{2}=\mathrm{OBn}, \mathrm{R}^{3}=\mathrm{OBn} \quad(21+24,72 \%)$

Scheme 2. Synthesis of the protected pentasaccharide derivatives.

\subsection{Transformation of the Protected Pentasaccharides into the End-Products}

Transformation of compounds 6-10 into the fully $O$-methylated and $O$-sulfated mono- and disulfonic acid end-products requires eight further synthetic steps including acetyl-, NAP- and benzyl-deprotections, formation of the glucuronic acid unit, liberation of carboxylic and sulfonic esters and the introduction of methyl ether and sulfate ester functions. We envisioned a reaction sequence in which the introduction of methyl ethers precedes the oxidative formation of the glucuronide residue 
E. To study the efficacy of this procedure, compound 9 was subjected to Zemplén deacetylation to liberate the hydroxyls to be methylated (Scheme 3). Upon deacetylation with $\mathrm{NaOMe}$, nucleophilic cleavage of the sulfonic-acid ester of unit $\mathbf{F}$ also occurred in some extent. Hence, the obtaining mixture of the sulfonate ester and sodium sulfonate derivatives was unified by treating with sodium iodide in acetone to give sulfonic acid salt 25 in an $81 \%$ yield over two steps. Introduction of the methyl ethers to the liberated hydroxyls was accomplished by alkylation using methyl iodide and sodium hydride to afford the desired $\mathbf{2 6}$ in a $67 \%$ yield. Subsequently, the 6-position of the penultimate glucose unit was unmasked by oxidative de-O-(2-napthyl)methylation to produce 27 in $80 \%$. TEMPO-BAIB mediated oxidation of $\mathbf{2 7}$ proceeded slowly and required large amounts of the co-oxidant BAIB to eventually produce, after $48 \mathrm{~h}$, the glucuronide derivative 28 , along with its partially debenzylated derivatives. Compound 28 could not be separated from the by-products, thus, this mixture was subjected to the remaining transformation, including basic hydrolysis of the iduronic ester, catalytic hydrogenolysis and $O$-sulfation to furnish 29 , a monosulfonic acid analogue of idraparinux.
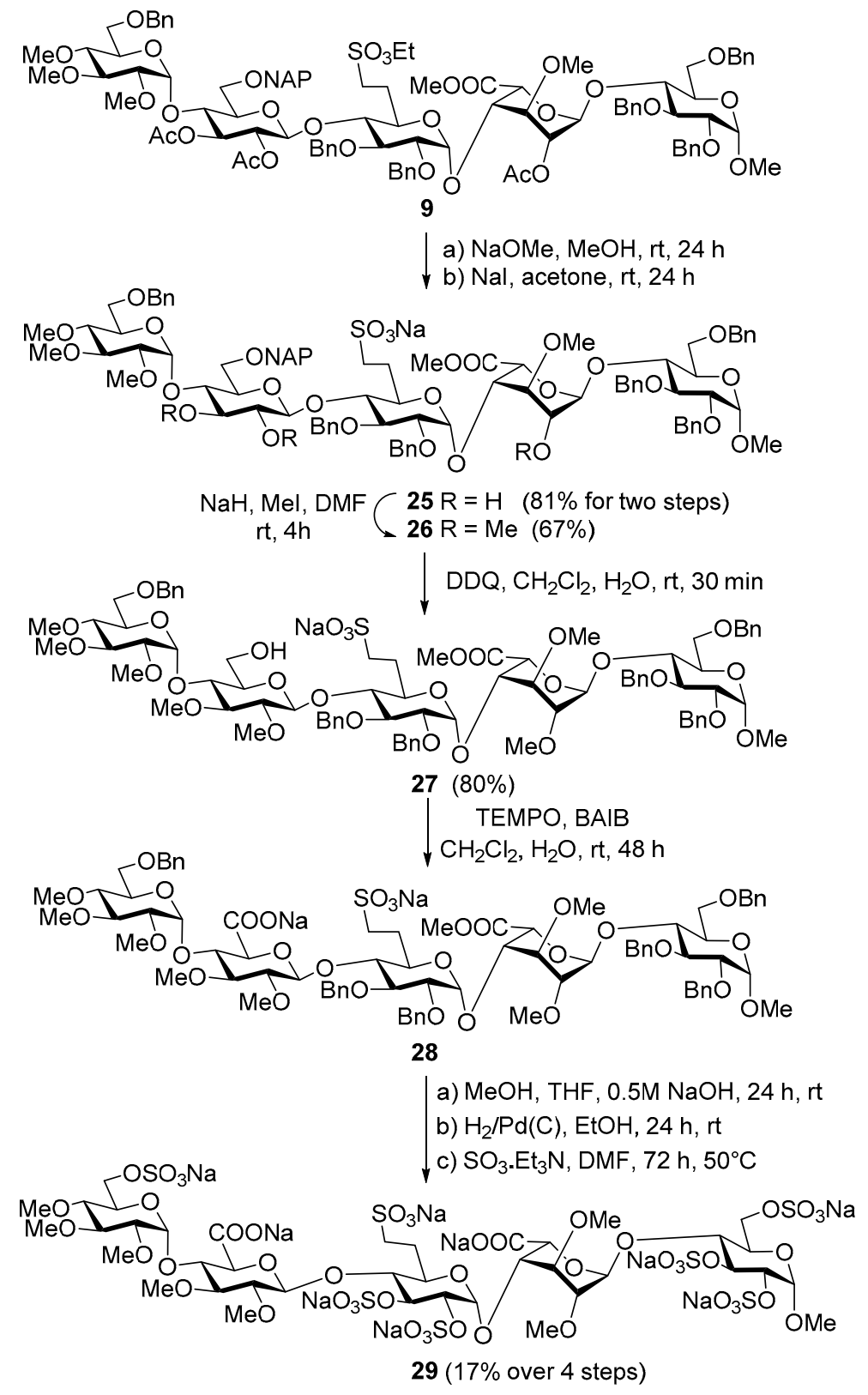

Scheme 3. Transformation of 9 into final product 29, a novel sulfonic acid analogue of idraparinux. 
For conversion of 6 into the final sulfated analogue, another reaction sequence, starting with the formation of the glucuronide residue, was used. The NAP protecting group of unit $\mathbf{E}$ was cleaved with DDQ to afford 30. Oxidation of the liberated 6-OH by TEMPO and BAIB proceeded smoothly to provide the required glucuronic acid-containing pentasaccharide 31 in $80 \%$. Zemplén deacetylation followed by deprotection of the sulfonic acid esters by nucleophilic displacement with sodium iodide gave the trisodium salt 32. Methylation of the free hydroxyl groups by using methyl iodide and sodium hydride afforded the desired 33, which possessed all of the required methyl ethers. Deprotection of the carboxylic-ester group of $\mathbf{3 3}$ by saponification gave tetrasodium salt $\mathbf{3 4}$, de-O-benzylation of which, by catalytic hydrogenolysis, furnished compound 35 in a high yield. O-Sulfation of the pentaol by using $\mathrm{SO}_{3} \cdot \mathrm{Et}_{3} \mathrm{~N}$ was surprisingly sluggish and the use of a high excess reagent and a prolonged reaction time were needed for completion of the sulfate ester formation. Finally, the reaction gave, after treatment with Dowex $\mathrm{Na}^{+}$ion-exchange resin, compound 36 as a new isosteric disulfonic acid analogue of idraparinux (Scheme 4).

The ${ }^{1} \mathrm{H}-\mathrm{NMR}$, and ${ }^{13} \mathrm{C}-\mathrm{NMR}$ spectra of the new compounds are reported in the Supplementary data.
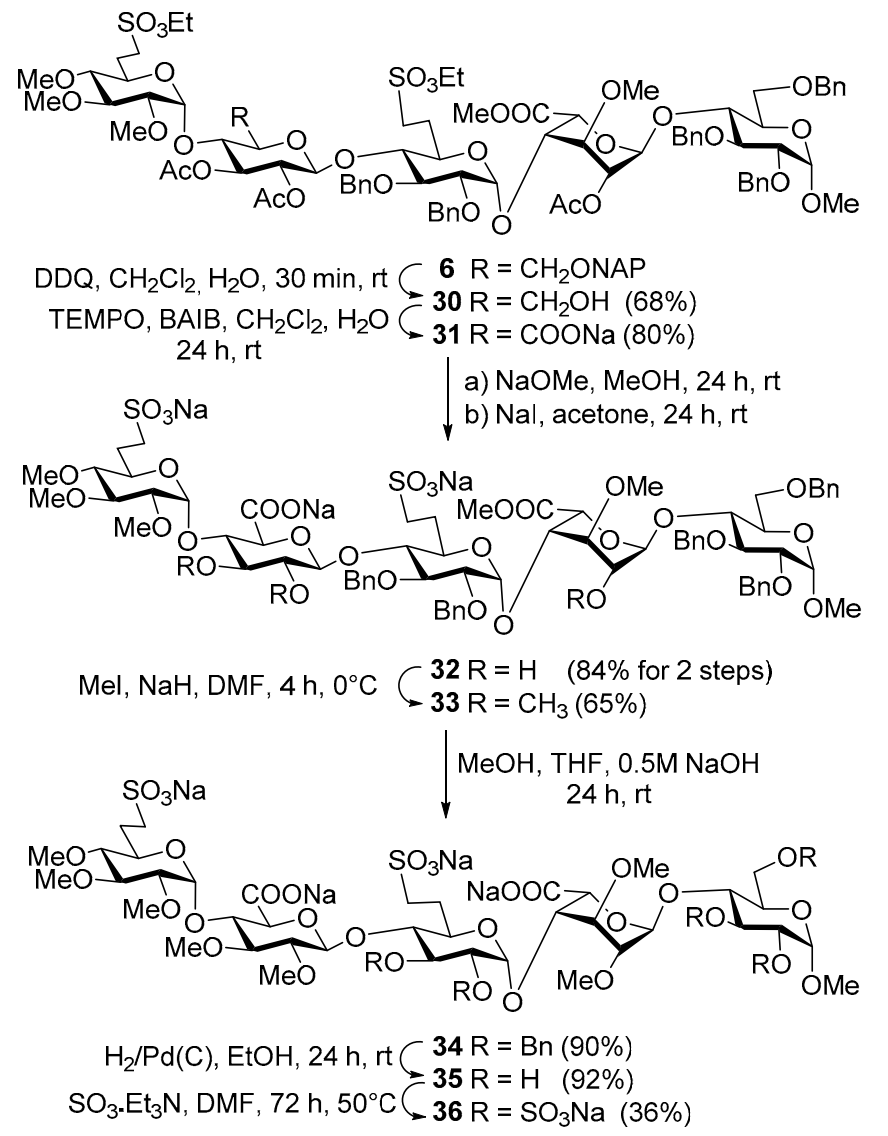

Scheme 4. Transformation of 6 into the corresponding pentasaccharide disulfonic acid final product.

\section{Materials and Methods}

\subsection{General Information}

Optical rotations were measured at room temperature with a Perkin-Elmer 241 automatic polarimeter. Thin layer chromatography (TLC) was performed on Kieselgel 60 F254 (Merck) with detection by immersing into $5 \%$ ethanolic sulfuric acid solution followed by heating. Column chromatography was performed on Silica gel 60 (Merck 0.063-0.200 mm). Organic solutions were dried over $\mathrm{MgSO}_{4}$, and concentrated in a vacuum. The ${ }^{1} \mathrm{H}-\mathrm{NMR}\left(360\right.$ and $400 \mathrm{MHz}$ ) and ${ }^{13} \mathrm{C}-\mathrm{NMR}$ (90.54 and 100.28 MHz) spectra were recorded with Bruker DRX-360 and DRX-400 spectrometers at 
$25{ }^{\circ} \mathrm{C}$. Chemical shifts are referenced to $\mathrm{Me}_{4} \mathrm{Si}$ or 4,4-dimethyl-4-silapentane-1-sulfonic acid (DSS) $\left(0.00 \mathrm{ppm}\right.$ for $\left.{ }^{1} \mathrm{H}\right)$ and to the solvent signals $\left(\mathrm{CDCl}_{3}: 77.00 \mathrm{ppm}\right.$ for $\left.{ }^{13} \mathrm{C}\right)$. The ${ }^{1} \mathrm{H}$ - and ${ }^{13} \mathrm{C}-\mathrm{NMR}$ assignments have been established from ${ }^{1} \mathrm{D}-\mathrm{NMR}$ spectra and for compounds 6, 7, 8, 9, 10, 20 and 21 the proton-signal assignments were supported by analysis of two-dimensional ${ }^{1} \mathrm{H}-{ }^{1} \mathrm{H}$ correlation spectra (COSY), as well as the carbon-signal assignments by two-dimensional ${ }^{13} \mathrm{C}-{ }^{1} \mathrm{H}$ correlation maps (HSQC). Infrared (IR) spectra were recorded on a Perkin-Elmer 16 PC FTIR (Program counter Fourier transform infrared) spectrometer. Matrix-assisted laser desorption/ionization-time-of-flight mass spectrometric (MALDI-TOF MS analyses of the compounds were carried out in the positive reflectron mode using a BIFLEX III mass 13 spectrometer (Bruker, Rheinstetten, Germany) equipped with delayed-ion extraction. The matrix solution was a saturated 2,4,6-trihydroxy-acetophenone (THAP) solution in MeCN. Elemental analyses $(\mathrm{C}, \mathrm{H}, \mathrm{S})$ were performed using an Elementar Vario MicroCube instrument.

\subsection{General Method A for TEMPO-BAIB Oxidation $(\mathbf{1 2}, \mathbf{2 8}, \mathbf{3 1})$}

To a vigorously stirred solution of the appropriate alcohol $(1 \mathrm{mmol})$ in $\mathrm{CH}_{2} \mathrm{Cl}_{2}(3.5 \mathrm{~mL})$ and $\mathrm{H}_{2} \mathrm{O}$ $(1.5 \mathrm{~mL})$, TEMPO $(0.2 \mathrm{mmol})$ and BAIB $(2 \mathrm{mmol})$ were added and the reaction mixture was stirred until TLC showed complete conversion of the starting material. The reaction time was $45 \mathrm{~min}$ for $\mathbf{1 2}$, $24 \mathrm{~h}$ for 31 and $48 \mathrm{~h}$ for 28 . The reaction mixture was quenched by the addition of $10 \%$ aq $\mathrm{Na}_{2} \mathrm{~S}_{2} \mathrm{O}_{3}$ solution $(20 \mathrm{~mL})$. The mixture was then extracted twice with EtOAc $(10 \mathrm{~mL})$, and the combined organic layers were dried, and concentrated.

\subsection{General Method B for Glycosylation Reaction Using NIS-TfOH Promoter System $(\mathbf{7}, \mathbf{1 7}, \mathbf{1 8}, \mathbf{1 9})$}

To a solution of donor $(1.5 \mathrm{mmol})$ and acceptor $(1 \mathrm{mmol})$ in dry $\mathrm{CH}_{2} \mathrm{Cl}_{2}(20 \mathrm{~mL}), 4 \AA$ molecular sieves were added. The stirred mixture was cooled to $-60^{\circ} \mathrm{C}(\mathbf{1 7}, \mathbf{1 8}, \mathbf{1 9})$ and $-50^{\circ} \mathrm{C}(7)$ under argon. After $30 \mathrm{~min}$ at this temperature, NIS $(2.25 \mathrm{mmol})$ and TfOH $(0.045 \mathrm{mmol})$ dissolved in THF $(155 \mu \mathrm{L})$ were added. The temperature was increased to $-50{ }^{\circ} \mathrm{C}(\mathbf{1 7}, \mathbf{1 8}, 19)$ or $-20^{\circ} \mathrm{C}(7)$. The reaction mixture was quenched with $\mathrm{Et}_{3} \mathrm{~N}(50 \mu \mathrm{L})$, diluted with $\mathrm{CH}_{2} \mathrm{Cl}_{2}(100 \mathrm{~mL})$ and the reaction mixture was extracted with saturated $\mathrm{Na}_{2} \mathrm{~S}_{2} \mathrm{O}_{3}$ solution $(20 \mathrm{~mL})$, saturated $\mathrm{NaHCO}_{3}$ solution $(20 \mathrm{~mL})$ and with distilled water $(20 \mathrm{~mL})$. The organic phase was dried and concentrated.

\subsection{General Method C for Glycosylation Using NIS-AgOTf Promoter System $\mathbf{6}, \mathbf{( 8 , 9 , 1 0 , 1 7 )}$}

To a solution of acceptor $(1 \mathrm{mmol})$ and donor $(1.5 \mathrm{mmol})$ in dry $\mathrm{CH}_{2} \mathrm{Cl}_{2}(20 \mathrm{~mL}), 4 \AA$ molecular sieves were added. The stirred mixture was cooled to between $-60{ }^{\circ} \mathrm{C}$ and $-35^{\circ} \mathrm{C}$ under argon. After $30 \mathrm{~min}$ at this temperature, $2.25 \mathrm{mmol}$ NIS dissolved in THF $(1 \mathrm{~mL})$ and $0.36 \mathrm{mmol} \mathrm{AgOTf}$ dissolved in toluene $(1 \mathrm{~mL})$ were added. After $1-4 \mathrm{~h}$ stirring at $-50{ }^{\circ} \mathrm{C}(\mathbf{1 7}),-20^{\circ} \mathrm{C}(\mathbf{9}),-15^{\circ} \mathrm{C}(\mathbf{6}),-10{ }^{\circ} \mathrm{C}(\mathbf{1 0})$ or $0{ }^{\circ} \mathrm{C}(8) \mathrm{Et}_{3} \mathrm{~N}(50 \mu \mathrm{L})$ was added. The reaction mixture was diluted with $\mathrm{CH}_{2} \mathrm{Cl}_{2}(100 \mathrm{~mL})$ and filtered through a pad of Celite. The filtrate was concentrated.

\subsection{General Method D for Removal of the (2-Naphthyl)methyl Ether Group (20, 21, 22, 27, 30)}

To a vigorously stirred solution of starting material $(1 \mathrm{mmol})$ in $\mathrm{CH}_{2} \mathrm{Cl}_{2}: \mathrm{H}_{2} \mathrm{O}(9: 1,10 \mathrm{~mL}) \mathrm{DDQ}$ (1.5 mmol) was added. The reaction mixture was stirred at room temperature for 30-40 min, diluted with $\mathrm{CH}_{2} \mathrm{Cl}_{2}(30 \mathrm{~mL})$, washed successively with satd aq $\mathrm{NaHCO}_{3}$ solution $(15 \mathrm{~mL})$ and $\mathrm{H}_{2} \mathrm{O}(15 \mathrm{~mL})$. The organic phase was dried and concentrated.

\subsection{Product Characterization}

Methyl [methyl (2-O-acetyl-3-O-methyl- $\alpha$-L-idopyranosyl)uronate](1 $\rightarrow 4)-2,3,4-t r i-O-b e n z y l-\alpha$-D-glucopyranoside (12). The starting material 11 [18] (3.20 g, $4.7 \mathrm{mmol})$ was oxidized according to general method A. The crude uronic acid was dissolved in THF $(4.5 \mathrm{~mL})$ and treated with ethereal diazomethane at $0{ }^{\circ} \mathrm{C}$. After complete disappearance of the uronic acid, the mixture was concentrated. The crude product 
was purified by column chromatography to give $12(2.47 \mathrm{~g}, 74 \%)$ as a colourless syrup; $R_{\mathrm{f}}=0.36$ (7:3 $n$-hexane/acetone); $[\alpha]_{\mathrm{D}}-2.67$ (c 1.00, $\left.\mathrm{CHCl}_{3}\right)$; IR $v_{\max }(\mathrm{KBr}): 3480,3474,3031,2936,2902,1744$, 1633, 1496, 1454, 1372, 1225, 1167, 1103, 1045, 911, 890, 854, 740, 700, $605 \mathrm{~cm}^{-1}$; ${ }^{1} \mathrm{H}-\mathrm{NMR}(360 \mathrm{MHz}$, $\left.\mathrm{CDCl}_{3}\right) \delta 7.37-7.20(\mathrm{~m}, 15 \mathrm{H}$, arom.), $5.08(\mathrm{~s}, 1 \mathrm{H}), 4.98(\mathrm{~d}, J=11.4 \mathrm{~Hz}, 2 \mathrm{H}), 4.87(\mathrm{~s}, 1 \mathrm{H}), 4.81(\mathrm{~d}, J=11.4 \mathrm{~Hz}$, $\left.1 \mathrm{H}, \mathrm{PhCH}_{2}\right), 4.67\left(\mathrm{~d}, J=12.0 \mathrm{~Hz}, 1 \mathrm{H}, \mathrm{PhCH}_{2}\right), 4.56-4.49(\mathrm{~m}, 4 \mathrm{H}), 3.99-3.80(\mathrm{~m}, 3 \mathrm{H}), 3.78-3.70(\mathrm{~m}, 2 \mathrm{H})$, 3.67-3.62 (m, 1H), $3.57(\mathrm{dd}, J=9.4,3.6 \mathrm{~Hz}, 1 \mathrm{H}), 3.50-3.44(\mathrm{~m}, 1 \mathrm{H}), 3.47,3.40,3.34\left(3 \mathrm{~s}, 9 \mathrm{H}, 3 \times \mathrm{CH}_{3}\right)$, $2.68(\mathrm{~d}, J=11.8 \mathrm{~Hz}, 1 \mathrm{H}, \mathrm{OH}), 2.01\left(\mathrm{~s}, 3 \mathrm{H}, \mathrm{COCH}_{3}\right) \mathrm{ppm} ;{ }^{13} \mathrm{C}-\mathrm{NMR}\left(91 \mathrm{MHz}, \mathrm{CDCl}_{3}\right) \delta 169.7,169.2$ $\left(\mathrm{COOCH}_{3}, \mathrm{COCH}_{3}\right), 139.0,138.0,138.0$ (3C, $\mathrm{C}_{\mathrm{q}}$ arom. $), 128.4,128.4,128.2,128.1,127.9,127.6,127.6$, 127.2, 127.0 (15C, arom.), 98.0, 97.6 (C-1-H, C-1-G), 80.3, 79.7, 76.8, 74.7, 70.1, 68.0, 67.2, 67.1 (C-2-G, C-2-H, C-3-G, C-3-H, C 4 G, C-4-H, C-5-G, C-5-H), 74.8, 73.4, $73.3\left(3 \times \mathrm{PhCH}_{2}\right), 68.5(\mathrm{C}-6-\mathrm{H}), 58.1$ $\left(\mathrm{OCH}_{3}, \mathrm{C}-3-\mathrm{G}\right), 55.2\left(\mathrm{OCH}_{3}, \mathrm{C}-1-\mathrm{H}\right), 51.9\left(\mathrm{COOCH}_{3}\right), 21.00\left(\mathrm{COCH}_{3}\right)$ ppm; MALDI-TOF MS: $\mathrm{m} / z$ z33.16 $[\mathrm{M}+\mathrm{Na}]^{+}$(Calcd. 733.28); Anal. Calcd. for $\mathrm{C}_{38} \mathrm{H}_{46} \mathrm{O}_{13}$ (710.29): C, 64.21; H, 6.52; O, 29.26. Found: C, 64.28; H, 6.55 .

Methyl [2,3-di-O-Benzyl-4-O-(2-naphthyl)methyl-6-deoxy-6-C-(ethylsulfonatomethyl)- $\alpha$-D-glucopyranosyl]$(1 \rightarrow 4)$-[methyl (2-O-acetyl-3-O-methyl- $\alpha$-L-idopyranosyl)uronate]-(1 $\rightarrow 4)-(2,3,6-t r i-O-b e n z y l-\alpha-$

D-glucopyranoside) (17)

I. To a solution of acceptor $12(2.80 \mathrm{~g}, 3.94 \mathrm{mmol})$ and donor 15 [16] (3.85 g, $5.91 \mathrm{mmol})$ in dry $\mathrm{CH}_{2} \mathrm{Cl}_{2}(20 \mathrm{~mL}), 4 \AA$ molecular sieves $(0.50 \mathrm{~g})$ were added. The stirred mixture was cooled to $-60^{\circ} \mathrm{C}$ under argon and activated by method $\mathrm{B}$. The reaction mixture was allowed to warm up to $-50{ }^{\circ} \mathrm{C}$ in $1 \mathrm{~h}$. The crude product was purified by column chromatography (7:3n-hexane/EtOAc) to give $\mathbf{1 7}$ $(2.35 \mathrm{~g}, 46 \%)$. Unreacted $12(1.11 \mathrm{~g}, 28 \%)$ was recovered as a colourless syrup.

II. To a solution of acceptor $12(1.11 \mathrm{~g}, 1.41 \mathrm{mmol})$ and donor $\mathbf{1 5}(1.37 \mathrm{~g}, 2.11 \mathrm{mmol})$ in dry $\mathrm{CH}_{2} \mathrm{Cl}_{2}$ (20 mL), $4 \AA$ molecular sieves $(0.50 \mathrm{~g})$ were added. The stirred mixture was cooled to $-60{ }^{\circ} \mathrm{C}$ under argon and activated by method $\mathrm{C}$. The reaction mixture was allowed to warm up to $-50^{\circ} \mathrm{C}$ for $1 \mathrm{~h}$. The crude product was purified by column chromatography (7:3 n-hexane/EtOAc) to give $\mathbf{1 7}$ (1.47 g, $73 \%)$ as a white foam; $R_{\mathrm{f}}=0.34(6: 4 n$-hexane $/ \mathrm{EtOAc}) ;[\alpha]_{\mathrm{D}}+11.30$ (c 0.81, $\left.\mathrm{CHCl}_{3}\right)$; IR $v_{\max }(\mathrm{KBr}): 3447$, 3087, 3061, 3030, 2933, 1763, 1737, 1636, 1604, 1497, 1455, 1369, 1357, 1238, 1169, 1107, 1028, 1002, 917, $857 \mathrm{~cm}^{-1} ;{ }^{1} \mathrm{H}-\mathrm{NMR}\left(360 \mathrm{MHz}, \mathrm{CDCl}_{3}\right) \delta 7.83-7.66$ (m, 4H, arom.), 7.49-7.42 (m, 2H, arom.), 7.39-7.16 (m, 26H, arom.), $5.13(\mathrm{~s}, 1 \mathrm{H}), 5.03\left(\mathrm{~d}, J=11.3 \mathrm{~Hz}, 1 \mathrm{H}, \mathrm{ArCH}_{2}\right), 4.93(\mathrm{~m}, 2 \mathrm{H}), 4.85-4.72(\mathrm{~m}, 7 \mathrm{H}), 4.66$ $(\mathrm{t}, J=12.6 \mathrm{~Hz}, 2 \mathrm{H}), 4.60-4.54(\mathrm{~m}, 3 \mathrm{H}), 4.12\left(\mathrm{q}, J=7.1 \mathrm{~Hz}, 2 \mathrm{H}, \mathrm{SO}_{3} \mathrm{CH}_{2} \mathrm{CH}_{3}\right), 3.96-3.61(\mathrm{~m}, 9 \mathrm{H}), 3.54$ $(\mathrm{dd}, J=9.4,3.5 \mathrm{~Hz}, 1 \mathrm{H}), 3.44-3.34(\mathrm{~m}, 1 \mathrm{H}), 3.41,3.36,3.34\left(3 \mathrm{~s}, 9 \mathrm{H}, 3 \times \mathrm{OCH}_{3}\right), 3.24-3.05(\mathrm{~m}, 3 \mathrm{H})$, 2.36-2.24 (m, 1H, H-7 $), 2.00-1.83\left(\mathrm{~m}, 4 \mathrm{H}, \mathrm{COCH}_{3}, \mathrm{H}-7_{\mathrm{b}}\right), 1.25\left(\mathrm{t}, J=7.1 \mathrm{~Hz}, 3 \mathrm{H}, \mathrm{SO}_{3} \mathrm{CH}_{2} \mathrm{CH}_{3}\right) \mathrm{ppm}$; ${ }^{13} \mathrm{C}-\mathrm{NMR}\left(91 \mathrm{MHz}, \mathrm{CDCl}_{3}\right) \delta 170.1,169.4(2 \times \mathrm{CO}), 139.1,138.4,138.1,138.1,138.1,135.6,133.3,133.0$ (8C, $C_{\mathrm{q}}$ arom.), 128.6, 128.5, 128.4, 128.4, 128.2, 128.2, 128.0, 127.9, 127.9, 127.7, 127.6, 127.3, 127.1, 126.5, 126.2, 126.0, 125.7 (32C, arom.), 98.7, 98.0, 97.7 (3 × C-1), 81.5, 81.2, 80.4, 80.2, 79.8, 76.5, 74.7, 74.7, 70.1, 69.5, 68.3, 67.7 (12C, skeleton carbons), 75.5, 75.1, 74.9, 73.6, 73.4, $73.4\left(6 \times \mathrm{ArCH}_{2}\right), 68.5(\mathrm{C}-6-\mathrm{F}), 66.2$ $\left(\mathrm{SO}_{3} \mathrm{CH}_{2} \mathrm{CH}_{3}\right), 58.3,55.2,51.8\left(3 \times \mathrm{OCH}_{3}\right), 46.5(\mathrm{C}-7-\mathrm{H}), 25.9(\mathrm{C}-6-\mathrm{H}), 21.1\left(\mathrm{COCH}_{3}\right), 15.1\left(\mathrm{SO}_{3} \mathrm{CH}_{2} \mathrm{CH}_{3}\right)$ ppm; MALDI-TOF MS: $m / z$ 1321.57 [M + Na] $]^{+}$(Calcd. 1321.50); Anal. Calcd. for $\mathrm{C}_{72} \mathrm{H}_{82} \mathrm{O}_{20} \mathrm{~S}(1298.51)$ : C, 66.55; H, 6.36; O, 24.62; S, 2.47. Found: C, 66.62; H, 6.40; S, 2.45.

Methyl [2,3,6-tri-O-benzyl-4-O-(2-naphthyl)methyl- $\alpha$-D-glucopyranosyl]-(1 $\rightarrow 4)$-[methyl (2-O-acetyl-3-O-methy$\alpha$-L-idopyranosyl)uronate]-(1 $\rightarrow 4)-2,3-d i-O-b e n z y l-6-d e o x y-6-C-(e t h y l s u l f o n a t o m e t h y l)-\alpha$-D-glucopyranoside 18. To a solution of acceptor (14) [17] (620 mg, $0.85 \mathrm{mmol})$ and donor 16 [17] ( $874 \mathrm{mg}, 1.28 \mathrm{mmol})$ in dry $\mathrm{CH}_{2} \mathrm{Cl}_{2}(20 \mathrm{~mL}) 4 \AA$ molecular sieves $(0.50 \mathrm{~g})$ were added. The stirred mixture was cooled to $-60^{\circ} \mathrm{C}$ under argon and activated by method $\mathbf{B}$. The reaction mixture was allowed to warm up to $-50{ }^{\circ} \mathrm{C}$ for $1 \mathrm{~h}$. The crude product was purified by column chromatography (7:3n-hexane/EtOAc) to give $\mathbf{1 8}$ $(1.07 \mathrm{~g}, 98 \%)$ as a white foam; $R_{\mathrm{f}}=0.63(1: 1 n$-hexane $/ \mathrm{EtOAc}) ;[\alpha]_{\mathrm{D}}+6.62\left(c 0.35, \mathrm{CHCl}_{3}\right) ; \mathrm{IR} v_{\max }$ (KBr): 3446, 3087, 3061, 3030, 2931, 2869, 1739, 1636, 1604, 1497, 1455, 1362, 1236, 1165, 1107, 1045, 1028, 1004, 917, 857, $818 \mathrm{~cm}^{-1}$; ${ }^{1} \mathrm{H}-\mathrm{NMR}\left(360 \mathrm{MHz}, \mathrm{CDCl}_{3}\right) \delta 7.85-7.67$ (m, 3H, arom.), 7.55 (s, $1 \mathrm{H}$, arom), 7.49-7.41 (m, 2H, arom.), 7.38-7.14 (m, 26H, arom.), 5.25 (s, 1H), 4.99-4.35 (m, 16H), 4.27 (q, J = 7.1 Hz, 
$\left.2 \mathrm{H}, \mathrm{SO}_{3} \mathrm{CH}_{2} \mathrm{CH}_{3}\right), 3.97-3.63(\mathrm{~m}, 7 \mathrm{H}), 3.62-3.24(\mathrm{~m}, 4 \mathrm{H}), 3.47,3.40,3.32\left(3 \times \mathrm{OCH}_{3}\right), 3.16-3.04(\mathrm{~m}, 1 \mathrm{H})$, 2.44-2.32 (m, 1H, H-7 $\mathrm{a}), 2.03\left(\mathrm{~s}, 3 \mathrm{H}, \mathrm{COCH}_{3}\right), 2.01-1.85\left(\mathrm{~m}, 1 \mathrm{H}, \mathrm{H}-7_{\mathrm{b}}\right), 1.37\left(\mathrm{t}, 3 \mathrm{H}, \mathrm{SO}_{3} \mathrm{CH}_{2} \mathrm{CH}_{3}\right) \mathrm{ppm}$; ${ }^{13} \mathrm{C}-\mathrm{NMR}\left(91 \mathrm{MHz}, \mathrm{CDCl}_{3}\right) \delta 170.2,169.6(2 \times \mathrm{CO}), 139.0,138.7,138.3,138.1,138.0,136.3,133.3,132.9$ (8C, $C_{\mathrm{q}}$ arom.), 128.5, 128.4, 128.2, 128.1, 128.0, 127.9, 127.7, 127.2, 126.1, 125.9, 125.8, 125.6 (32C, arom.), 99.6, 98.0, $98.0(3 \times \mathrm{C}-1), 81.8,80.3,80.0,79.5,78.6,77.0,75.1,71.4,69.2,68.4,68.1,57.9$ (12C, skeleton carbons), 75.5, 75.2, 74.7, 73.5, 73.5, $73.4\left(6 \times \mathrm{ArCH}_{2}\right), 68.1(\mathrm{C}-6-\mathrm{H}), 66.2\left(\mathrm{SO}_{3} \mathrm{CH}_{2} \mathrm{CH}_{3}\right), 58.7,55.6,51.9$ $\left(3 \times \mathrm{OCH}_{3}\right), 46.7(\mathrm{C}-7-\mathrm{F}), 26.0(\mathrm{C}-6-\mathrm{F}), 21.1\left(\mathrm{COCH}_{3}\right), 15.2\left(\mathrm{SO}_{3} \mathrm{CH}_{2} \mathrm{CH}_{3}\right) \mathrm{ppm}$; MALDI-TOF MS: $\mathrm{m} / z$ 1321.57 [M + Na] ${ }^{+}$(Calcd. 1321.50); Anal. Calcd. for $\mathrm{C}_{72} \mathrm{H}_{82} \mathrm{O}_{20} \mathrm{~S}$ (1298.51): C, 66.55; H, 6.36; O, 24.62; S, 2.47. Found: C, 66.50; H, 6.42; S, 2.51.

Methyl [2,3,6-tri-O-benzyl-4-O-(2-naphthyl)methyl- $\alpha$-D-glucopyranosyl]-(1 $\rightarrow 4)$-[methyl (2-O-acetyl-3-O-methyl$\alpha$-L-idopyranosyl)uronate]-(1 $\rightarrow 4)-(2,3,6$-tri-O-benzyl- $\alpha$-D-glucopyranoside) (19). To a solution of acceptor $12(1.50 \mathrm{~g}, 2.10 \mathrm{mmol})$ and donor $16(2.16 \mathrm{~g}, 3.16 \mathrm{mmol})$ in dry $\mathrm{CH}_{2} \mathrm{Cl}_{2}(20 \mathrm{~mL}) 4 \AA$ molecular sieves $(0.50 \mathrm{~g})$ were added. The stirred mixture was cooled to $-60^{\circ} \mathrm{C}$ under argon and activated by method $\mathbf{B}$. The reaction mixture was allowed to warm up to $-50{ }^{\circ} \mathrm{C}$ for $30 \mathrm{~min}$. The crude product was purified by column chromatography (7:3 $n$-hexane/EtOAc) to give $18(2.41 \mathrm{~g}, 80 \%)$ as a white foam. $R_{\mathrm{f}}=0.47$ (6:4 $n$-hexane/EtOAc).

Methyl [2,3-di-O-benzyl-6-deoxy-6-C-(ethylsulfonatomethyl)- $\alpha$-D-glucopyranosyl]-(1 $\rightarrow 4)-[m e t h y l ~(2-O-a c e t y l-3-$ O-methyl- $\alpha$-L-idopyranosyl)uronate]-( $1 \rightarrow 4)-(2,3,6-$ tri-O-benzyl- $\alpha$-D-glucopyranoside) (20). Compound 17 $(2.50 \mathrm{~g}, 1.92 \mathrm{mmol})$ was converted to 20 according to general method $\mathbf{D}$. The crude product was purified by column chromatography (7:3 $n$-hexane/EtOAc) to give compound $20(1.55 \mathrm{~g}, 70 \%)$ as a white foam; $R_{\mathrm{f}}=0.40$ (1:1 n-hexane/EtOAc); $[\alpha]_{\mathrm{D}}+13.46$ (c 0.56, $\left.\mathrm{CHCl}_{3}\right) ; \mathrm{IR} v_{\max }(\mathrm{KBr}): 3502,3088$, 3063, 3031, 2933, 1738, 1629, 1497, 1455, 1370, 1356, 1236, 1168, 1108, 1047, 1028, 917, 820, 740, 698, 606, 545, $466 \mathrm{~cm}^{-1} ;{ }^{1} \mathrm{H}-\mathrm{NMR}\left(400 \mathrm{MHz}, \mathrm{CDCl}_{3}\right) \delta 7.38-7.19$ (m, 25H, arom.), 5.15 (s, 1H, H-1-G), 4.93 (d, $\left.J=11.4 \mathrm{~Hz}, 2 \mathrm{H}, \mathrm{BnCH}_{2}\right), 4.85-4.76\left(\mathrm{~m}, 4 \mathrm{H}, \mathrm{H}-2-\mathrm{G}, \mathrm{H}-5-\mathrm{G}, \mathrm{H}-1-\mathrm{F}, \mathrm{BnCH}_{2}\right), 4.75-4.61\left(\mathrm{~m}, 4 \mathrm{H}, \mathrm{BnCH}_{2}\right)$, $4.55\left(\mathrm{~m}, 3 \mathrm{H}, \mathrm{H}-1-\mathrm{H}, \mathrm{BnCH}_{2}\right), 4.24\left(\mathrm{q}, J=7.1 \mathrm{~Hz}, 2 \mathrm{H}, \mathrm{SO}_{3} \mathrm{CH}_{2} \mathrm{CH}_{3}\right), 3.94-3.79(\mathrm{~m}, 3 \mathrm{H}, \mathrm{H}-3-\mathrm{H}, \mathrm{H}-4-\mathrm{G})$, 3.77-3.59 (m, 6H, H-3-G, H-3-F, H-5-F, H-4-H, H-6 $\mathrm{a}_{\mathrm{b}}-\mathrm{H}$ ), 3.54 (dd, J = 9.3, 3.5 Hz, 1H, H-2-H), 3.43-3.31 (m, 2H, H-2-F, H-5-H), 3.40, 3.37, 3.34 (3s, $\left.3 \times \mathrm{CH}_{3}\right)$ 3.28-3.09 (m, 3H, H-4-F, H-7 a, ), $2.48(\mathrm{~s}, 1 \mathrm{H}, \mathrm{OH})$, 2.33-2.21 (m, 1H, H-6 $\left.6_{\mathrm{a}}-\mathrm{F}\right), 1.93\left(\mathrm{~s}, 3 \mathrm{H}, \mathrm{COCH}_{3}\right), 1.98-1.84\left(\mathrm{~m}, 1 \mathrm{H}, \mathrm{H}-6_{\mathrm{b}}-\mathrm{F}\right), 1.35$ (t, $J=7.1 \mathrm{~Hz}, 3 \mathrm{H}$, $\left.\mathrm{SO}_{3} \mathrm{CH}_{2} \mathrm{CH}_{3}\right) \mathrm{ppm} ;{ }^{13} \mathrm{C}-\mathrm{NMR}\left(101 \mathrm{MHz}, \mathrm{CDCl}_{3}\right) \delta 170.0,169.3(2 \times \mathrm{CO}), 139.1,138.5,138.1,138.0$, 137.9 (5C, $C_{\mathrm{q}}$ arom.), 128.6, 128.5, 128.4, 128.3, 128.1, 128.1, 128.0, 127.9, 127.8, 127.8, 127.7, 127.5, 127.3, 127.1 (25C, arom.), 98.5 (C-1-F), 98.0 (C-1-H), 97.7 (C-1-G), 80.6 (C-3-F), 80.1 (C-2-H), 79.9 (C-2-F), 79.8 (C-3-H), 79.8 (C-4-G), 76.5 (C-5-F), 74.8 (C-2-G), $74.4(\mathrm{C}-4-\mathrm{F}), 75.0,74.9,73.4,73.3,73.2\left(5 \times \mathrm{PhCH}_{2}\right)$, $70.1(\mathrm{C}-4-\mathrm{H}), 69.8$ (C-5-H), $68.5(\mathrm{C}-3-\mathrm{F}), 68.4(\mathrm{C}-6-\mathrm{H}), 68.0(\mathrm{C}-5-\mathrm{G}), 66.2\left(\mathrm{SO}_{3} \mathrm{CH}_{2} \mathrm{CH}_{3}\right), 58.4,55.2,51.7$ $\left(3 \times \mathrm{OCH}_{3}\right), 46.3(\mathrm{C}-7-\mathrm{F}), 25.8(\mathrm{C}-6-\mathrm{F}), 21.0\left(\mathrm{COCH}_{3}\right), 15.1\left(\mathrm{SO}_{3} \mathrm{CH}_{2} \mathrm{CH}_{3}\right) \mathrm{ppm}$; MALDI-TOF MS: $\mathrm{m} / z$ $1181.59[\mathrm{M}+\mathrm{Na}]^{+}$(Calcd. 1181.44); Anal. Calcd. for $\mathrm{C}_{61} \mathrm{H}_{74} \mathrm{O}_{20} \mathrm{~S}$ (1158.45): C, 63.20; $\mathrm{H}, 6.43 ; \mathrm{O}, 27.60$; S, 2.77. Found: C, 63.25; H, 6.37; S, 2.72.

Methyl [2,3,6-tri-O-benzyl- $\alpha$-D-glucopyranosyl]-(1 $\rightarrow 4)$-[methyl(2-O-acetyl-3-O-methyl- $\alpha$-L-idopyranosyl)uronate](1 $\rightarrow 4)$-2,3-di-O-benzyl-6-deoxy-6-C-(ethylsulfonatomethyl)- $\alpha$-D-glucopyranoside (21). Compound 18 (1.06 g, $0.83 \mathrm{mmol}$ ) was converted to 21 according to general method $\mathbf{D}$. The crude product was purified by column chromatography (65:35 $\mathrm{n}$-hexane/acetone) to give compound $\mathbf{2 1}(807 \mathrm{mg}, 85 \%)$ as a white foam; $R_{\mathrm{f}}=0.38$ (65:35 $n$-hexane/acetone); $[\alpha]_{\mathrm{D}}+14.44\left(\right.$ c $\left.0.04, \mathrm{CHCl}_{3}\right) ; \mathrm{IR} v_{\max }(\mathrm{KBr}): 3481,3063$, 3031, 2929, 1740, 1626, 1497, 1455, 1370, 1234, 1167, 1105, 1046, 1028, 926, 820, 739, 698, 606, 548, 460, $418 \mathrm{~cm}^{-1}$; ${ }^{1} \mathrm{H}-\mathrm{NMR}\left(400 \mathrm{MHz}, \mathrm{CDCl}_{3}\right) 87.30-7.08$ (m, 25H, arom.), 5.17 (d, $\left.J=3.3 \mathrm{~Hz}, 1 \mathrm{H}, \mathrm{H}-1-\mathrm{G}\right)$, 4.86-4.65 (m, 3H, H-1-F, H-2-G, H-5-G, BnCH 2 ), 4.59 (m, 3H, BnCH $)$, 4.50-4.34 (m, 4H, H-1-H, BnCH ( $_{2}$, $4.18\left(\mathrm{q}, J=7.1 \mathrm{~Hz}, 2 \mathrm{H}, \mathrm{SO}_{3} \mathrm{CH}_{2} \mathrm{CH}_{3}\right), 3.85-3.14(\mathrm{~m}, 13 \mathrm{H}$, skeleton protons), 3.36, 3.29, $3.23(3 \mathrm{~s}, 9 \mathrm{H}$, $\left.\mathrm{OCH}_{3}\right), 3.05-2.95\left(\mathrm{~m}, 1 \mathrm{H}, \mathrm{H}-7_{\mathrm{b}}-\mathrm{H}\right), 2.46(\mathrm{~s}, 1 \mathrm{H}, \mathrm{OH}), 2.34-2.24\left(\mathrm{~m}, 1 \mathrm{H}, \mathrm{H}-6_{\mathrm{a}}-\mathrm{H}\right), 1.93\left(\mathrm{~s}, 3 \mathrm{H}, \mathrm{COCH}_{3}\right)$, 1.89-1.76 (m, $\left.1 \mathrm{H}, \mathrm{H}-6_{\mathrm{b}}-\mathrm{H}\right), 1.28\left(\mathrm{t}, J=7.1 \mathrm{~Hz}, 3 \mathrm{H}, \mathrm{SO}_{3} \mathrm{CH}_{2} \mathrm{CH}_{3}\right) \mathrm{ppm} ;{ }^{13} \mathrm{C}-\mathrm{NMR}\left(101 \mathrm{MHz}, \mathrm{CDCl}_{3}\right) \delta$ 170.2, $169.6(2 \times \mathrm{CO}), 139.0,138.8,138.2,138.1,138.0$ (5C, $C_{\mathrm{q}}$ arom.), 128.6, 128.5, 128.4, 128.2, 128.0, 127.9, 127.8, 127.8, 127.3 (25C, arom.), 99.5 (C-1-F), 98.1 (C-1-H), 98.0 (C-1-G), 81.1, 80.2, 79.5, 79.5, 78.9, 
77.0, 75.0, 71.2, 70.9, 69.8 (12C, skeleton carbons), 75.3, 75.1,73.7, 73.6, $73.3\left(5 \times \mathrm{PhCH}_{2}\right), 69.3(\mathrm{C}-6-\mathrm{F})$, 69.0, 68.2, $66.3\left(\mathrm{SO}_{3} \mathrm{CH}_{2} \mathrm{CH}_{3}\right), 58.9,55.6,51.9\left(3 \times \mathrm{OCH}_{3}\right), 46.8(\mathrm{C}-7-\mathrm{H}), 25.9(\mathrm{C}-6-\mathrm{H}), 21.1\left(\mathrm{COCH}_{3}\right)$, $15.2\left(\mathrm{SO}_{3} \mathrm{CH}_{2} \mathrm{CH}_{3}\right)$ ppm; MALDI-TOF MS: $m / z 1181.64[\mathrm{M}+\mathrm{Na}]^{+}$(Calcd. 1181.44); Anal. Calcd. for $\mathrm{C}_{61} \mathrm{H}_{74} \mathrm{O}_{20} \mathrm{~S}$ (1158.45): C, 63.20; H, 6.43; O, 27.60; S, 2.77. Found: C, 63.34; H, 6.47; S, 2.79.

Methyl [2,3,6-tri-O-benzyl- $\alpha$-D-glucopyranosyl]-(1 $\rightarrow 4)$-[methyl-(2-O-acetyl-3-O-methyl- $\alpha$-L-idopyranosyl)uronate]$(1 \rightarrow 4)$-(2,3,6-tri-O-benzyl- $\alpha$-D-glucopyranoside) (22) [15]. Compound 19 (2.4 g, $1.87 \mathrm{mmol})$ was converted to 22 according to general method $\mathbf{D}$. The crude product was purified by column chromatography (7:3 $n$-hexane/EtOAc) to give compound $22(1.26 \mathrm{~g}, 59 \%)$ as a white foam; $R_{\mathrm{f}}=0.33$ (6:4 n-hexane/EtOAc); $[\alpha]_{\mathrm{D}}+8.41\left(c 0.62, \mathrm{CHCl}_{3}\right)\left(\right.$ lit. [15] $[\alpha]_{\mathrm{D}}+2.3\left(c \mathrm{c} .10, \mathrm{CHCl}_{3}\right) ; \mathrm{IR} v_{\max }(\mathrm{KBr}): 3087,3062,3031,2932,2906$, $1739,1605,1497,1455,1371,1237,1104,1046,1028,908,738,697,606,538,459,419 \mathrm{~cm}^{-1}$. The NMR spectroscopic and analytical data of 22 are consistent with those given in the literature [15].

Methyl [2,3,4-tri-O-methyl-6-deoxy-6-C-(ethylsulfonatomethyl)- $\alpha$-D-glucoopiranosyl]-(1 $\rightarrow 4)-[2,3-d i-O-a c e t y l-6-$ O-(2-naphthyl)methyl- $\beta$-D-glucopyranosyl]-(1 $\rightarrow 4)-[2,3-d i-O-$-benzyl-6-deoxy-6-C-(ethylsulfonatomethyl)- $\alpha$ D-glucopyranosyl]-(1 $\rightarrow 4)$-[methyl (2-O-acetyl-3-O-methyl- $\alpha$-L-idopyranosyl) uronate]-(1 $\rightarrow 4)-2,3,6$-tri-Obenzyl- $\alpha$-D-gluco-pyranoside (6). To a solution of acceptor 20 (630 mg, $0.54 \mathrm{mmol}$ ) and donor 23 $(660 \mathrm{mg}, 0.82 \mathrm{mmol})$ in dry $\mathrm{CH}_{2} \mathrm{Cl}_{2}(20 \mathrm{~mL}), 4 \AA$ molecular sieves $(0.50 \mathrm{~g})$ were added. The stirred mixture was cooled to $-40{ }^{\circ} \mathrm{C}$ under argon and activated by method $\mathrm{C}$. The reaction mixture was allowed to warm up to $-15^{\circ} \mathrm{C}$ for $4 \mathrm{~h}$. The crude product was purified by column chromatography (6:4 $n$-hexane/EtOAc) to give $6(626 \mathrm{mg}, 62 \%)$ as a colourless syrup; $R_{\mathrm{f}}=0.26(1: 1 n$-hexane/EtOAc); $[\alpha]_{\mathrm{D}}+23.69\left(c_{0} 0.14, \mathrm{CHCl}_{3}\right) ;{ }^{1} \mathrm{H}-\mathrm{NMR}\left(400 \mathrm{MHz}, \mathrm{CDCl}_{3}\right) \delta$ 7.84-7.75 (m, 3H, arom.), 7.65 (s, 1H, arom.), 7.48-7.40 (m, 2H, arom.), 7.37-7.13 (m, 26H, arom.), 5.18 (t, J = 9.2 Hz, 1H, H-3-E), 5.11 (s, 1H, H-1-G), 4.99-4.46 (m, 19H, H-1-D, H-2-E, H-5-G, H-2-G, H-1-E, H-1-H, H-1-F, $\left.12 \times \mathrm{ArCH}_{2}\right), 4.27,4.07$ (2q, $4 \mathrm{H}, \mathrm{SO}_{3} \mathrm{CH}_{2} \mathrm{CH}_{3}$ ), 3.93-3.77 (m, 5H, H-4-H, H-4-E, H-3-F, H-4-G, H-3-H), 3.76-3.59 (m, 5H, H-5-H, H-5-F, H-6 $\left.{ }_{\mathrm{a}, \mathrm{b}}-\mathrm{H}, \mathrm{H}-3-\mathrm{G}\right), 3.58-3.17$ (m, 9H, H-2-F, H-6 $\left.{ }_{\mathrm{a}, \mathrm{b}}-\mathrm{E}, \mathrm{H}-5-\mathrm{D}, \mathrm{H}-4-\mathrm{F}, \mathrm{H}-2-\mathrm{H}, \mathrm{H}-3-\mathrm{D}, \mathrm{H}-5-\mathrm{E}, \mathrm{H}-7 \mathrm{a}-\mathrm{F}\right)$, $3.52,3.48,3.40,3.36,3.33,3.31\left(6 \mathrm{~s}, 18 \mathrm{H}, 6 \times \mathrm{OCH}_{3}\right), 3.18-3.03(\mathrm{~m}, 2 \mathrm{H}, \mathrm{H}-7 \mathrm{~b}-\mathrm{F}, \mathrm{H}-7 \mathrm{a}-\mathrm{D}), 2.93-2.81$ (m, 2H, H-2-D, H-7b-D), 2.65 (t, $J=9.2 \mathrm{~Hz}, 1 \mathrm{H}, \mathrm{H}-4-\mathrm{D}), 2.33-2.21\left(\mathrm{~m}, 1 \mathrm{H}, \mathrm{H}-6_{\mathrm{a}}-\mathrm{F}\right), 2.21-2.08$ (m, 1H, H-6 $\left.{ }_{\mathrm{a}}-\mathrm{D}\right), 2.05,1.99,1.94\left(3 \mathrm{~s}, 9 \mathrm{H}, 3 \times \mathrm{COCH}_{3}\right), 1.86-1.71\left(\mathrm{~m}, 2 \mathrm{H}, \mathrm{H}-6_{\mathrm{b}}-\mathrm{D}, \mathrm{H}-6_{\mathrm{b}}-\mathrm{F}\right), 1.38,(\mathrm{t}, J=7.1 \mathrm{~Hz}, 3 \mathrm{H}$, $\left.\mathrm{SO}_{3} \mathrm{CH}_{2} \mathrm{CH}_{3}\right), 1.25\left(\mathrm{t}, \mathrm{J}=6.8 \mathrm{~Hz}, 3 \mathrm{H}, \mathrm{SO}_{3} \mathrm{CH}_{2} \mathrm{CH}_{3}\right) \mathrm{ppm} ;{ }^{13} \mathrm{C}-\mathrm{NMR}\left(101 \mathrm{MHz}, \mathrm{CDCl}_{3}\right) \delta 170.1,169.8$, $169.8,169.3(4 \times \mathrm{CO}), 139.1,138.9,138.0,137.9,137.7,135.3,133.2,132.9\left(8 \mathrm{C}, \mathrm{C}_{\mathrm{q}}\right.$ arom. $), 128.4,128.3$, $128.2,128.1,128.0,128.0,127.9,127.8,127.8,127.6,127.5,127.1,127.0,126.4,126.1,125.8,125.6(32 \mathrm{C}$, arom.), 101.1 (C-1-H), 98.0 (C-1-E), 97.8 (C-1-F), 97.4 (C-1-G), 96.8 (C-1-D), 83.2 (C-4-D), 82.3 (C-3-D), 82.1 (C-4-F), 81.9 (C-2-D), 80.0 (C-2-F), 79.7 (C-2-H), 79.6 (C-4-G), 79.4 (C-3-F), 76.0 (C-3-G), 75.1 (C-5-E), 74.7, 74.3, 73.6, 73.4, 73.2, 73.2, $\left(6 \times \mathrm{ArCH}_{2}\right), 74.5$ (C-3-E), $74.5(\mathrm{C}-4-\mathrm{H}), 74.4$ (C-4-E), $74.0(\mathrm{C}-3-\mathrm{H}), 72.5$ (C-2-E), 70.0 (C-5-H), 69.2 (C-5-D), 68.9 (C-5-F), 68.3 (C-6-H), 68.2 (C-5-G), 67.7 (C-6-E), 67.3 (C-2-G), 66.2, $65.8\left(2 \times \mathrm{SO}_{3} \mathrm{CH}_{2} \mathrm{CH}_{3}\right), 60.5,60.5,58.9,58.1,55.1,51.7\left(6 \times \mathrm{OCH}_{3}\right), 46.6$ (C-7-D), 46.4 (C-7-F), 26.0 (C-6-D), 25.7 (C-6-F), 21.0, 20.8, $20.5\left(3 \times \mathrm{COCH}_{3}\right), 15.0,15.0\left(2 \times \mathrm{SO}_{3} \mathrm{CH}_{2} \mathrm{CH}_{3}\right)$ ppm; MALDI-TOF MS: $m / z$ 1877.77 [M + Na] ${ }^{+}$(Calcd. 1877.68); Anal. Calcd. for $\mathrm{C}_{94} \mathrm{H}_{118} \mathrm{O}_{34} \mathrm{~S}_{2}$ (1854.69): C, 60.83; $\mathrm{H}, 6.41 ; \mathrm{O}$, 29.31; S, 3.46. Found: C, 60.69; H, 6.35; S, 3.41.

Methyl [2,3,4-tri-O-methyl-6-deoxy-6-C-(ethylsulfonatomethyl)- $\alpha$-D-glucopyranosyl]-(1 $\rightarrow 4)-[2,3-d i-O-a c e t y l-6-$ O-(2-naphthyl)methyl- $\beta$-D-glucopyranosyl]-(1 $\rightarrow 4)-[2,3,6-t r i-O-b e n z y l-\alpha$-D-glucopyranosyl]-(1 $\rightarrow 4)-[m e t h y l$ (2-O-acetyl-3-O-methyl- $\alpha$-L-idopyranosyl)uronate]-(1 $\rightarrow 4)-2,3-$ di-O-benzyl-6-deoxy-6-C-(ethylsulfonatomethyl)$\alpha$-D-glucopyranoside (7). To a solution of acceptor $21(720 \mathrm{mg}, 0.62 \mathrm{mmol})$ and donor 23 (752 mg, $0.93 \mathrm{mmol})$ in dry $\mathrm{CH}_{2} \mathrm{Cl}_{2}(20 \mathrm{~mL}) 4 \AA$ molecular sieves $(0.50 \mathrm{~g})$ were added. The stirred mixture was cooled to $-50{ }^{\circ} \mathrm{C}$ under argon and activated by method $\mathbf{B}$. The reaction mixture was allowed to warm up to $-20{ }^{\circ} \mathrm{C}$ for $3 \mathrm{~h}$. The crude product was purified by column chromatography $(65: 35$ $n$-hexane/EtOAc) to give $7(726 \mathrm{mg}, 63 \%)$ as a colourless syrup; $R_{\mathrm{f}}=0.21\left(1: 1 n\right.$-hexane $/$ EtOAc); $[\alpha]_{\mathrm{D}}$ +32.21 (c 0.14, $\left.\mathrm{CHCl}_{3}\right) ;{ }^{1} \mathrm{H}-\mathrm{NMR}\left(400 \mathrm{MHz}, \mathrm{CDCl}_{3}\right) \delta 7.83-7.71$ (m, 3H, arom.), 7.62 (s, 1H, arom.), 7.44 (m, 2H, arom.), 7.41-7.12 (m, 26H, arom.), 5.21 (d, J = 2.3 Hz, 1H, H-1-G), 5.10-4.99 (m, 1H, H-3-E, $\mathrm{ArCH}_{2}$ ), 4.96-4.76 (m, 6H, H-1-D, H-2-G, H-2-E, H-1-F, $2 \times \mathrm{ArCH}_{2}$ ), 4.75-4.63 (m, 5H, H-5-G, 
$\left.4 \times \mathrm{ArCH}_{2}\right), 4.60-4.38\left(\mathrm{~m}, 7 \mathrm{H}, \mathrm{H}-1-\mathrm{E}, \mathrm{H}-1-\mathrm{H}, 5 \times \mathrm{ArCH}_{2}\right), 4.29\left(\mathrm{q}, J=7.1 \mathrm{~Hz}, 2 \mathrm{H}, \mathrm{SO}_{3} \mathrm{CH}_{2} \mathrm{CH}_{3}\right), 4.06$ $\left(\mathrm{q}, J=7.1 \mathrm{~Hz}, 2 \mathrm{H}, \mathrm{SO}_{3} \mathrm{CH}_{2} \mathrm{CH}_{3}\right), 3.95(\mathrm{t}, J=9.4 \mathrm{~Hz}, 1 \mathrm{H}, \mathrm{H}-4-\mathrm{H}), 3.91-3.67\left(\mathrm{~m}, 8 \mathrm{H}, \mathrm{H}-4-\mathrm{G}, \mathrm{H}-4-\mathrm{E}, \mathrm{H}-\mathrm{a}_{\mathrm{a}}-\mathrm{E}\right.$, H-5-H, H-3-H, H-3-F, H-6 $\left.{ }_{\mathrm{a}}-\mathrm{F}, \mathrm{H}-5-\mathrm{F}\right), 3.67-3.25$ (m, 9H, H-3-G, H-6 $-\mathrm{b}, \mathrm{H}, \mathrm{6}_{\mathrm{b}}-\mathrm{E}, \mathrm{H}-4-\mathrm{F}, \mathrm{H}-5-\mathrm{D}, \mathrm{H}-2-\mathrm{H}$, H-2-F, H-3-D, H-7-H), 3.57, 3.53, 3.43, 3.41, 3.33 (5s, 15H, $\left.5 \times \mathrm{OCH}_{3}\right), 3.23-3.04$ (m, 3H, H-5-E, H-7a-D, $\mathrm{H}-7 \mathrm{a}-\mathrm{H}), 2.97-2.87$ (m, 2H, H-2-D, H-7 $\mathrm{b}-\mathrm{D}), 2.69$ (t, J = 9.2 Hz, 1H, H-4-D), 2.44-2.30 (m, 1H, H-6 $\mathrm{a}-\mathrm{H})$, 2.30-2.14 (m, 1H, H-6 $\left.\mathrm{a}_{\mathrm{a}}-\mathrm{D}\right), 2.02,1.96,1.95\left(3 \mathrm{~s}, 9 \mathrm{H}, 3 \times \mathrm{COCH}_{3}\right), 2.06-1.74\left(\mathrm{~m}, 2 \mathrm{H}, \mathrm{H}-6_{\mathrm{b}}-\mathrm{D}, \mathrm{H}-6_{\mathrm{b}}-\mathrm{H}\right)$, $1.40\left(\mathrm{t}, J=7.1 \mathrm{~Hz}, 3 \mathrm{H}, \mathrm{SO}_{3} \mathrm{CH}_{2} \mathrm{CH}_{3}\right), 1.23\left(\mathrm{t}, J=7.4 \mathrm{~Hz}, 3 \mathrm{H}, \mathrm{SO}_{3} \mathrm{CH}_{2} \mathrm{CH}_{3}\right)$ ppm. ${ }^{13} \mathrm{C}-\mathrm{NMR}(101 \mathrm{MHz}$, $\left.\mathrm{CDCl}_{3}\right) \delta 170.3,170.0,169.7,169.6(4 \times \mathrm{CO}), 139.4,139.0,138.3,138.1,137.6,135.8,133.4,133.0(8 \mathrm{C}$, $\mathrm{C}_{\mathrm{q}}$ arom.), 128.8, 128.6, 128.5, 128.4, 128.4, 128.2, 128.2, 128.1, 128.0, 127.9, 127.8, 127.6, 127.3, 126.4, 126.1, 125.9, 125.8 (32C, arom.), 99.8 (C-1-E), 99.6 (C-1-F), 98.0 (C-1-H), 97.8 (C-1-G), 96.9 (C-1-D), 83.5 (C-4-D), 82.7 (C-3-D), 82.2 (C-2-D), 80.3 (C-4-F), 79.7 (C-3-H), 79.4 (C-3-F), 79.3 (C-2-F), 78.6 (C-2-H), 76.7 (C-4-H), 76.5 (C-3-G), 75.3 (C-5-E), 74.9 (C-4-G), 74.9 (C-3-E), 74.9 (C-4-E), 72.5 (C-2-E), 71.1 (C-5-F), 69.5 (C-5-D), 69.3 (C-5-G), 68.2 (C-5-H), 68.2 (C-2-G), 75.3, 75.1, 73.9, 73.7, 73.6, $73.6\left(6 \times \mathrm{ArCH}_{2}\right), 68.1$ (C-6-E), 67.4 (C-6-F), 66.3, $66.1\left(2 \times \mathrm{SO}_{3} \mathrm{CH}_{2} \mathrm{CH}_{3}\right), 60.8,60.8,59.2,58.8,55.6,52.0\left(6 \times \mathrm{OCH}_{3}\right), 46.8,46.8$ (C-7-D, C-7-H), 26.2 (C-6-D), $26.0(\mathrm{C}-6-\mathrm{H}), 21.1,21.0,20.8\left(3 \times \mathrm{COCH}_{3}\right), 15.2,15.1\left(2 \times \mathrm{SO}_{3} \mathrm{CH}_{2} \mathrm{CH}_{3}\right)$ ppm; MALDI-TOF MS: $m / z$ 1877.77 [M + Na] ${ }^{+}$(Calcd. 1877.68); Anal. Calcd. for $\mathrm{C}_{94} \mathrm{H}_{118} \mathrm{O}_{34} \mathrm{~S}_{2}$ (1854.69): C, 60.83; H, 6.41; O, 29.31; S, 3.46. Found: C, 60.90; H, 6.44; S, 3.51.

Methyl [2,3,4-tri-O-methyl-6-deoxy-6-C-(ethylsulfonatomethyl)- $\alpha$-D-glucopyranosyl]-(1 $\rightarrow 4)-[2,3-$ di-O-acetyl6-O-6-O-(2-naphthyl)methyl- $\beta$-D-glucopyranosyl]-(1 $\rightarrow 4)-[2,3,6$-tri-O-benzyl- $\alpha$-D-glucopyranosyl]-(1 $\rightarrow 4)$ [methyl-(2-O-acetyl-3-O-methyl- $\alpha$-L-idopyranosyl)uronate]-(1 $\rightarrow 4)-(2,3,6-$ tri-O-benzyl- $\alpha$-D-glucopyranoside) (8). To a solution of acceptor $22(1.18 \mathrm{~g}, 1.03 \mathrm{mmol})$ and donor $23(1.25 \mathrm{~g}, 1.55 \mathrm{mmol})$ in dry $\mathrm{CH}_{2} \mathrm{Cl}_{2}$ $(40 \mathrm{~mL}), 4 \AA$ molecular sieves $(1 \mathrm{~g})$ were added. The stirred mixture was cooled to $-50^{\circ} \mathrm{C}$ under argon and activated by method $\mathrm{C}$. The reaction mixture was allowed to warm up to $0{ }^{\circ} \mathrm{C}$ for $4 \mathrm{~h}$. The crude product was purified by column chromatography ( $6: 4 n$-hexane/EtOAc) to give $8(1.29 \mathrm{~g}, 68 \%)$ as a colourless syrup; $R_{\mathrm{f}}=0.51\left(94: 6 \mathrm{CH}_{2} \mathrm{Cl}_{2}\right.$ /acetone); $[\alpha]_{\mathrm{D}}+24.44\left(c 0.05, \mathrm{CHCl}_{3}\right) ;{ }^{1} \mathrm{H}-\mathrm{NMR}(400 \mathrm{MHz}$, $\left.\mathrm{CDCl}_{3}\right) \delta 7.86-7.71(\mathrm{~m}, 3 \mathrm{H}$, arom.), $7.62(\mathrm{~s}, 1 \mathrm{H}$, arom.), $7.44(\mathrm{~m}, 2 \mathrm{H}$, arom.), 7.40-7.11 (m, 31H, arom.), 5.15 (d, $J=2.7$ Hz, 1H, H-1-G), 5.05 (m, 2H, H-3-E, ArCH A $_{2}, 4.95-4.75$ (m, 7H, H-1-D, H-2-E, H-1-F, $\left.\mathrm{H}-2-\mathrm{G}, 3 \times \mathrm{ArCH}_{2}\right), 4.74-4.65\left(\mathrm{~m}, 5 \mathrm{H}, \mathrm{H}-5-\mathrm{G}, 4 \times \mathrm{ArCH}_{2}\right), 4.62-4.46\left(\mathrm{~m}, 6 \mathrm{H}, \mathrm{H}-1-\mathrm{H}, 5 \times \mathrm{ArCH}_{2}\right)$, 4.45-4.40 (m, 2H, H-1-E, $\left.\mathrm{ArCH}_{2}\right), 4.05\left(\mathrm{q}, J=7.1 \mathrm{~Hz}, 2 \mathrm{H}, \mathrm{SO}_{3} \mathrm{CH}_{2} \mathrm{CH}_{3}\right), 3.95(\mathrm{t}, J=9.4 \mathrm{~Hz}, 1 \mathrm{H}, \mathrm{H}-4-\mathrm{F})$, 3.91-3.45 (m, 16H, H-4-G, H-4-H, H-4-E, H-3-H, H-5-F, H-6 ${ }_{\mathrm{a}, \mathrm{b}}-\mathrm{H}, \mathrm{H}-3-\mathrm{F}, \mathrm{H}-5-\mathrm{H}, \mathrm{H}-3-\mathrm{G}, \mathrm{H}-\mathrm{a}_{\mathrm{a}, \mathrm{b}}$-E, H-6 $\left.a_{\mathrm{a}, \mathrm{b}}-\mathrm{F}, \mathrm{H}-5-\mathrm{D}, \mathrm{H}-2-\mathrm{H}\right), 3.57,3.53,3.41,3.35,3.34,3.32\left(6 \mathrm{~s}, 18 \mathrm{H}, 6 \times \mathrm{OCH}_{3}\right), 3.44-3.27$ (m, 4H, H-2-F, H-3-D, H-5-E, H-7a-D), 2.97-2.85 (m, 2H, H-2-D, H-7b-D), 2.69 (t, J = 9.2 Hz, 1H, H-4-D), 2.26-2.15 (m, $\left.1 \mathrm{H}, \mathrm{H}-6_{\mathrm{a}}-\mathrm{D}\right), 2.01,1.91,1.89$ (3s, 9H, $\left.3 \times \mathrm{COCH}_{3}\right), 1.87-1.75\left(\mathrm{~m}, 1 \mathrm{H}, \mathrm{H}-6_{\mathrm{b}}-\mathrm{D}\right), 1.23(\mathrm{t}, J=7.1 \mathrm{~Hz}, 3 \mathrm{H}$, $\left.\mathrm{SO}_{3} \mathrm{CH}_{2} \mathrm{CH}_{3}\right) \mathrm{ppm} ;{ }^{13} \mathrm{C}-\mathrm{NMR}\left(91 \mathrm{MHz}, \mathrm{CDCl}_{3}\right) \delta 170.2,170.0,169.7,169.6(4 \times \mathrm{CO}), 139.5,139.2,138.3$, 138.3 138.1, 137.7, 135.8, 133.4, 133.0 (9C, $C_{\mathrm{q}}$ arom.), 128.8, 128.5, 128.4, 128.2, 128.2, 128.0, 127.9, 127.8, 127.8, 127.6, 127.5, 127.3, 127.1, 126.4, 126.1, 125.9, 125.8 (37C, arom.), 99.8 (C-1-E), 99.7 (C-1-F), 98.1 (C-1-H), 97.8 (C-1-G), 96.9 (C-1-D), 83.6 (C-4-D), 82.7 (C-3-D), 82.2 (C-2-D), 80.1 (C-2-H), 79.9 (C-3-H), 79.8 (C-3-F), 79.3 (C-2-F), 76.8 (C-3-G), 76.5 (C-4-F), 75.3 (C-5-E), 75.1 (C-3-E), 75.1 (C-4-E), 74.9 (C-4-G), 74.9 (C-4-H), 75.1, 75.1, 75.1, 73.8, 73.6, 73.5, 73.5 (7 $\left.\times \mathrm{ArCH}_{2}\right), 72.5$ (C-2-E), 71.0 (C-5-F), $70.2(\mathrm{C}-5-\mathrm{H})$, 69.5 (C-5-D), 69.2 (C-5-G), 68.5 (C-2-G), 68.3 (C-6-F), 68.3 (C-6-E), 67.4 (C-6-H), $66.0\left(\mathrm{SO}_{3} \mathrm{CH}_{2} \mathrm{CH}_{3}\right)$, 60.8, 60.8, 59.2, 58.8, 55.3, $51.8\left(6 \times \mathrm{OCH}_{3}\right), 46.8(\mathrm{C}-7-\mathrm{D}), 26.2(\mathrm{C}-6-\mathrm{D}), 21.1,21.1,20.8\left(3 \times \mathrm{COCH}_{3}\right)$, $15.1\left(\mathrm{SO}_{3} \mathrm{CH}_{2} \mathrm{CH}_{3}\right)$ ppm; MALDI-TOF MS: $\mathrm{m} / z$ 1862.75 [M + Na] ${ }^{+}$(Calcd. 1863.02); Anal. Calcd. for $\mathrm{C}_{98} \mathrm{H}_{118} \mathrm{O}_{32} \mathrm{~S}$ (1840.03): $\mathrm{C}, 63.97 ; \mathrm{H}, 6.46 ; \mathrm{O}, 27.82 ; \mathrm{S}, 1.74$. Found: $\mathrm{C}, 64.03 ; \mathrm{H}, 6.49 ; \mathrm{S}, 1.68$.

Methyl-[6-O-benzyl-2,3,4-tri-O-methyl- $\alpha$-D-glucopyranosyl]-(1 $\rightarrow 4)$-[2,3-di-O-acetyl-6-O-(2-naphthyl)methyl$\beta$-D-glucopyranosyl]-(1 $\rightarrow 4)$-[2,3-di-O-benzyl-6-deoxy-6-C-(ethylsulfonatomethyl)- $\alpha$-D-glucopyranosyl]-(1 $\rightarrow 4)$ [methyl-(2-O-acetyl-3-O-methyl- $\alpha$-L-idopyranosyl)uronate]-(1 $\rightarrow 4)-(2,3,6$-tri-O-benzyl- $\alpha$-D-glucopyranoside) (9). To a solution of acceptor $20(1.20 \mathrm{~g}, 1.04 \mathrm{mmol})$ and donor $24(1.23 \mathrm{~g}, 1.55 \mathrm{mmol})$ in dry $\mathrm{CH}_{2} \mathrm{Cl}_{2}$ $(40 \mathrm{~mL}), 4 \AA$ molecular sieves $(1 \mathrm{~g})$ were added. The stirred mixture was cooled to $-35{ }^{\circ} \mathrm{C}$ under argon and activated by method $\mathrm{C}$. The reaction mixture was allowed to warm up to $-20^{\circ} \mathrm{C}$ for $4 \mathrm{~h}$. 
The crude product was purified twice by column chromatography (I. 6:4 $n$-hexane/EtOAc II. 96:4 $\left.\mathrm{CH}_{2} \mathrm{Cl}_{2} / \mathrm{EtOAc}\right)$ to give $9(1.20 \mathrm{~g}, 63 \%)$ as colourless; $R_{\mathrm{f}}=0.33\left(1: 1 n\right.$-hexane/EtOAc); $[\alpha]_{\mathrm{D}}+26.28$ (c $\left.0.06, \mathrm{CHCl}_{3}\right) ;{ }^{1} \mathrm{H}-\mathrm{NMR}\left(400 \mathrm{MHz}, \mathrm{CDCl}_{3}\right) \delta 7.84-7.71$ (m, 3H, arom.), 7.63 (s, $1 \mathrm{H}$, arom.), 7.50-7.41 (m, 2H, arom.), 7.38-7.11 (m, 31H, arom), $5.22(\mathrm{t}, J=9.3 \mathrm{~Hz}, 1 \mathrm{H}, \mathrm{H}-3-\mathrm{E}), 5.12-5.03(\mathrm{~m}, 2 \mathrm{H}, \mathrm{H}-1-\mathrm{G}$, H-1-D), $4.95\left(\mathrm{t}, J=12.3 \mathrm{~Hz}, 2 \mathrm{H}, 2 \times \mathrm{ArCH}_{2}\right), 4.89-4.48$ (m, 15H, H-2-E, H-5-G, H-2-G, H-1-E, H-1-F, $\left.\mathrm{H}-1-\mathrm{H}, 9 \times \mathrm{ArCH}_{2}\right), 4.40\left(\mathrm{~d}, J=10.9 \mathrm{~Hz}, 2 \mathrm{H}, 2 \times \mathrm{ArCH}_{2}\right), 4.26\left(\mathrm{~m}, 3 \mathrm{H}, \mathrm{SO}_{3} \mathrm{CH}_{2} \mathrm{CH}_{3}, \mathrm{ArCH}_{2}\right), 3.98-3.43$ (m, 14H, H-4-E, H-4-H, H-4-G, H-3-F, H-3-H, H-5-F, H-5-H, H-6a, b-H, H-3-G, H-6a, b-E, H-2-H, H-5-D), 3.55, 3.39, 3.39, 3.38, 3.34, 3.30 (6s, $\left.18 \mathrm{H}, 6 \times \mathrm{OCH}_{3}\right)$ 3.43-2.99 (m, 9H, H-6a, $-\mathrm{D}, \mathrm{H}-4-\mathrm{F}, \mathrm{H}-5-\mathrm{E}, \mathrm{H}-2-\mathrm{F}$, H-3-D, H-7 a,b-F, H-4-D, H-2-D), 2.31-2.20 (m, 1H, H-6a-F), 2.04, 1.99, 1.93 (3s, 9H, $\left.3 \times \mathrm{OCH}_{3}\right), 1.85-1.70$ $\left(\mathrm{m}, 1 \mathrm{H}, \mathrm{H}-\mathrm{b}_{\mathrm{b}}-\mathrm{F}\right), 1.39\left(\mathrm{t}, J=7.1 \mathrm{~Hz}, 3 \mathrm{H}, \mathrm{SO}_{3} \mathrm{CH}_{2} \mathrm{CH}_{3}\right) \mathrm{ppm} ;{ }^{13} \mathrm{C}-\mathrm{NMR}\left(91 \mathrm{MHz}, \mathrm{CDCl}_{3}\right) \delta 170.3,170.0$, $169.9,169.5(4 \times C O), 139.2,139.1,138.2,138.1,138.1,137.9,136.0,133.3,133.0$ (9C, $C_{\mathrm{q}}$ arom.), 128.5, $128.5,128.4,128.4,128.3,128.2,128.1,128.0,127.8,127.7,127.3,127.2,126.7,126.2,126.1,125.9,125.6$ (37C, arom.), 101.1 (C-1-E), 98.3 (C-1-F), 98.1 (C-2-H), 97.9 (C-1-D), 97.6 (C-1-G), 83.2 (C-3-D), 82.1 (C-4-F), 81.8 (C-2-D), 80.2 (C-2-H), 79.9 (C-3-H), 79.8 (C-2-F), 79.5 (C-3-F), 79.3 (C-4-D), 76.1 (C-3-G), 75.1 (C-5-E), 75.0 (C-3-E), 74.8 (C-4-H), 74.5 (C-4-E), 74.2 (C-4-G), 74.9, 74.6, 73.8, 73.5, 73.4, 73.4, 73.4 $\left(7 \times \mathrm{ArCH}_{2}\right), 72.9$ (C-2-E), $71.2(\mathrm{C}-5-\mathrm{D}), 70.2(\mathrm{C}-5-\mathrm{F}), 69.1$ (C-5-H), 68.6 (C-6-E), 68.5 (C-6-H), 68.4 (C-5-G), 68.3 (C-6-D), 67.4 (C-2-G), $66.3\left(\mathrm{SO}_{3} \mathrm{CH}_{2} \mathrm{CH}_{3}\right), 60.7,60.4,59.4,58.2,55.3,51.9\left(6 \times \mathrm{OCH}_{3}\right), 46.6$ (C-7-F), 25.9 (C-6-F), 21.2, 21.0, $20.7\left(3 \times \mathrm{COCH}_{3}\right), 15.2\left(\mathrm{SO}_{3} \mathrm{CH}_{2} \mathrm{CH}_{3}\right) \mathrm{ppm}$; MALDI-TOF MS: $\mathrm{m} / z$ 1862.74 [M + Na] $]^{+}$(Calcd. 1863.02); Anal. Calcd. for $\mathrm{C}_{98} \mathrm{H}_{118} \mathrm{O}_{32} \mathrm{~S}$ (1840.03): C, 63.97; H, 6.46; O, 27.82; S, 1.74. Found: C, 63.95; H, 6.51; S, 1.78 .

Methyl [6-O-benzyl-2,3,4-tri-O-methyl- $\alpha$-D-glucopyranosyl]-(1 $\rightarrow$ 4)-[2,3-di-O-acetyl-6-O-(2-naphthyl)methyl$\beta$-D-glucopyranosyl]-(1 $\rightarrow 4)$-[2,3,6-tri-O-benzyl- $\alpha$-D-glucopyranosyl]-(1 $\rightarrow 4)$-[methyl-(2-O-acetyl-3-O-methyl$\alpha$-L-idopyranosyl)uronate]-(1 $\rightarrow 4)$-2,3-di-O-benzyl-6-deoxy-6-C-(ethylsulfonatomethyl)- $\alpha$-D-glucopyranoside (10). To a solution of acceptor 21. (720 mg, $0.62 \mathrm{mmol})$ and donor $24(737 \mathrm{mg}, 0.93 \mathrm{mmol})$ in dry $\mathrm{CH}_{2} \mathrm{Cl}_{2}(20 \mathrm{~mL}), 4 \AA$ molecular sieves $(0.50 \mathrm{~g})$ were added. The stirred mixture was cooled to $-40^{\circ} \mathrm{C}$ under argon and activated by method $\mathrm{C}$. The reaction mixture was allowed to warm up to $-10^{\circ} \mathrm{C}$ for $90 \mathrm{~min}$. The crude product was purified twice by column chromatography (I. 9:1 $\mathrm{CH}_{2} \mathrm{Cl}_{2} / \mathrm{EtOAc}$ II. 6:4 $n$-hexane/EtOAc) to give $\mathbf{1 0}(825 \mathrm{mg}, 72 \%)$ as a colourless syrup; $R_{\mathrm{f}}=0.31$ (1:1 $n$-hexane/EtOAc); $[\alpha]_{\mathrm{D}}+35.45\left(c\right.$ $\left.0.05, \mathrm{CHCl}_{3}\right) ;{ }^{1} \mathrm{H}-\mathrm{NMR}\left(400 \mathrm{MHz}, \mathrm{CDCl}_{3}\right) \delta 7.82-7.69(\mathrm{~m}, 3 \mathrm{H}$, arom.), $7.62(\mathrm{~s}, 1 \mathrm{H}$, arom.), 7.48-7.09 (m, 30H, arom.), 5.21 (d, J = 5.0 Hz, 1H, H-1-G), 5.12-5.03 (m, 3H, H-3-E, H-1-D, ArCH , $^{2}$ 4.92-4.76 (m, 5H, H-2-E, H-1-F, H-2-G, $\left.2 \times \mathrm{ArCH}_{2}\right), 4.75-4.61\left(\mathrm{~m}, 5 \mathrm{H}, \mathrm{H}-5-\mathrm{G}, 4 \times \mathrm{ArCH}_{2}\right), 4.59-4.51$ $\left(\mathrm{m}, 3 \mathrm{H}, 3 \times \mathrm{ArCH}_{2}\right), 4.49-4.37\left(\mathrm{~m}, 5 \mathrm{H}, \mathrm{H}-1-\mathrm{E}, \mathrm{H}-1-\mathrm{H}, 3 \times \mathrm{ArCH}_{2}\right), 4.34-4.25\left(\mathrm{~m}, 3 \mathrm{H}, \mathrm{SO}_{3} \mathrm{CH}_{2} \mathrm{CH}_{3}\right.$, $\left.\mathrm{ArCH}_{2}\right), 4.01-3.84$ (m, 3H, H-4-H, H-4-E, H-4-G), 3.83-3.67 (m, 7H, H-3-H, H-5-F, H-6a-F, H-6a, $-\mathrm{E}$, H-5-H, H-3-F), 3.66-3.52 (m, 3H, H-3-G, H-5-D, H-6b-F), 3.50-3.26 (m, 7H, H-6a,b-D, H-2-H, H-4-F, H-3-D, H-2-F, H-7a-H), 3.59, 3.44, 3.42, 3.41, 3.33, 3.32 (6s, 18H, $\left.6 \times \mathrm{OCH}_{3}\right), 3.26-3.02$ (m, 4H, H-5-E, H-4-D, H-7a-H, H-2-D), 2.42-2.32 (m, 1H, H-6a-H), 2.01, 1.96, 1.94 (3s, 9H, $\left.3 \times \mathrm{COCH}_{3}\right), 1.98-1.93$ (m, $\left.1 \mathrm{H}, \mathrm{H}-7_{\mathrm{b}}-\mathrm{H}\right) 1.39\left(\mathrm{t}, J=12.7 \mathrm{~Hz}, 3 \mathrm{H}, \mathrm{SO}_{3} \mathrm{CH}_{2} \mathrm{CH}_{3}\right) \mathrm{ppm} ;{ }^{13} \mathrm{C}-\mathrm{NMR}\left(101 \mathrm{MHz}, \mathrm{CDCl}_{3}\right) \delta 170.3,170.0$, 169.7, $169.6(4 \times \mathrm{CO}), 139.4,139.0,138.4,138.1,138.1,137.7,136.2,133.4,133.0$ (9C, $C_{\mathrm{q}}$ arom.), 128.8, 128.6, 128.4, 128.4, 128.3, 128.2, 128.1, 128.0, 127.8, 127.7, 127.6, 127.3, 127.2, 126.0, 125.9, 125.7 (37C, arom.), 99.8 (C-1-E), 99.6 (C-1-F), 98.0 (C-1-H), 97.9 (C-1-G), 97.9 (C-1-D), 83.3 (C-3-D), 81.9 (C-2-D), 80.3 (C-4-F), 79.8 (C-5-H), 79.8 (C-3-F), 79.4 (C-4-D), 79.4 (C-3-H), 79.2 (C-2-F), 78.6 (C-2-H), 76.7 (C-3-G), 76.5 (C-4-H), 75.1 (C-4-G), 75.1 (C-4-E), 75.1 (C-5-E) 74.9 (C-3-E), 75.3, 75.1, 73.9, 73.7, 73.6, 73.4, $73.4\left(7 \times \mathrm{ArCH}_{2}\right), 72.7$ (C-2-E), 71.4 (C-5-D), 71.1 (C-5-F), 69.3 (C-5-G), 68.9(C-6-E), 68.6 (C-6-D), 68.2 (C-2-G), 67.4 (C-6-F), $66.2\left(\mathrm{SO}_{3} \mathrm{CH}_{2} \mathrm{CH}_{3}\right), 60.8,60.5,59.3,58.8,55.6,51.9\left(6 \times \mathrm{OCH}_{3}\right), 46.8(\mathrm{C}-7-\mathrm{H}), 26.1$ (C-6-H), 21.1, 21.0, $20.8\left(3 \times \mathrm{COCH}_{3}\right), 15.2\left(\mathrm{SO}_{3} \mathrm{CH}_{2} \mathrm{CH}_{3}\right)$ ppm; ESI-MS: $m / z 1862.75[\mathrm{M}+\mathrm{Na}]^{+}$(Calcd. 1863.02); Anal. Calcd. for $\mathrm{C}_{98} \mathrm{H}_{118} \mathrm{O}_{32} \mathrm{~S}$ (1838,73): C, 63.97; H, 6.46; O, 27.82; S, 1.74. Found: C, 64.07; $\mathrm{H}, 6.53 ; \mathrm{S}, 1.82$.

Methyl [6-O-benzyl-2,3,4-tri-O-methyl- $\alpha$-D-glucopyranosyl]-(1 $\rightarrow 4)-[6-O-(2-n a p h t h y l)-m e t h y l-\beta-D-$ glucopyranosyl]-(1 $\rightarrow 4)$-[sodium (2,3-di-O-benzyl-6-deoxy-6-C-sulfonato-methyl- $\alpha$-D-glucopyranosyl)]-(1 $\rightarrow 4)$ - 
[methyl (3-O-methyl- $\alpha$-L-idopyranosyl)uronate]-(1 $\rightarrow 4)-(2,3,6-t r i-O-b e n z y l-\alpha-D-g l u c o p y r a n o s i d e)$

(25). $\mathrm{NaOMe}(36 \mathrm{mg})$ was added to the solution of compound $9(1.22,0.66 \mathrm{mmol})$ in $\mathrm{MeOH}(35 \mathrm{~mL})$ at room temperature and stirred for $24 \mathrm{~h}$. The reaction mixture was quenched by the addition of acetic acid (1-2 drops) and the solution was concentrated. Then, NaI (149 mg, $0.99 \mathrm{mmol}$ ) was added to the solution of crude product in acetone $(40 \mathrm{~mL})$ and the mixture was stirred at room temperature for $24 \mathrm{~h}$. The mixture was concentrated and purified by gel chromatography (Sephadex LH-20, MeOH) to give 25 (919 $\mathrm{mg}, 81 \%$ for two steps) as a colourless syrup; $R_{\mathrm{f}}=0.28\left(95: 5 \mathrm{CH}_{2} \mathrm{Cl}_{2} / \mathrm{MeOH}\right) ;[\alpha]_{\mathrm{D}}$

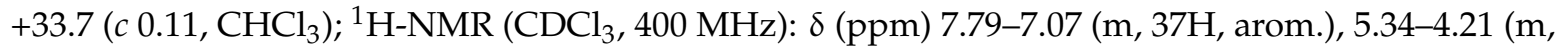
$\left.19 \mathrm{H}, 7 \times \mathrm{ArCH}_{2}, 5 \times \mathrm{H}-1\right), 3.98-2.87\left(\mathrm{~m}, 49 \mathrm{H}, 20 \times\right.$ skeleton protons, $6 \times \mathrm{OCH}_{3}, 3 \times \mathrm{H}-6_{\mathrm{a}, \mathrm{b}}, \mathrm{H}-7_{\mathrm{a}, \mathrm{b}}$, $3 \times \mathrm{OH}), 2.43-2.38\left(\mathrm{~m}, 1 \mathrm{H}, \mathrm{H}-6_{\mathrm{a}}-\mathrm{F}\right), 2.13-2.03\left(\mathrm{~m}, 1 \mathrm{H}, \mathrm{H}-6_{\mathrm{b}}-\mathrm{F}\right) ;{ }^{13} \mathrm{C}-\mathrm{NMR}\left(\mathrm{CDCl}_{3}, 100 \mathrm{MHz}\right): \delta(\mathrm{ppm})$ 171.3 (CO), 140.7, 140.1, 139.4, 139.3, 138.9, 137.4, 134.6, 134.3 (9 × $\mathrm{C}_{\mathrm{q}}$ arom.), 129.4-126.8 (37C, arom.), 104.3, 102.2, 99.5, 98.9, $96.0(5 \times \mathrm{C}-1), 84.6,83.5,81.7,81.3,81.2,80.7,80.3,79.7,78.0,76.3,75.9,75.6$, 72.6, 72.3, 71.6, 70.8, 69.2, 67.9 (20C, skeleton carbons), 75.7, 74.9, 74.4, 74.2, $73.9\left(7 \times \mathrm{ArCH}_{2}\right), 69.8$ $(3 \times \mathrm{C}-6) 60.9,60.8,59.8,58.5\left(4 \times \mathrm{OCH}_{3}\right), 55.5\left(\mathrm{C}-1-\mathrm{OCH}_{3}\right), 52.9\left(\mathrm{COOCH}_{3}\right), 48.2(\mathrm{C}-7-\mathrm{F}), 27.6(\mathrm{C}-6-\mathrm{F})$; MALDI-TOF MS: $m / z$ 1729.72 [M + Na] ${ }^{+}$(Calcd. 1729.64). Anal. Calcd. for $\mathrm{C}_{90} \mathrm{H}_{107} \mathrm{NaO}_{29} \mathrm{~S}(1707.85)$ : C, 63.29; H, 6.31; S, 1.88. Found: C, 63.37; H, 6.40; S, 1.97.

Methyl [6-O-benzyl-2,3,4-tri-O-methyl- $\alpha$-D-glucopyranosyl]-(1 $\rightarrow 4)-[2,3-d i-O-m e t h y l-6-O-(2-n a p h t h y l) m e t h y l-$ $\beta$-D-glucopyranosyl]-(1 $\rightarrow 4)$-[sodium (2,3-di-O-benzyl-6-deoxy-6-C-sulfonatomethyl- $\alpha$-D-glucopyranosyl)]$(1 \rightarrow 4)$-[methyl (2,3-di-O-methyl- $\alpha$-L-idopyranosyl)uronate]-(1 $\rightarrow 4)-(2,3,6$-tri-O-benzyl- $\alpha$-D-glucopyranoside) (26). An amount of $60 \mathrm{~m} / \mathrm{m} \% \mathrm{NaH}(68 \mathrm{mg}, 1.68 \mathrm{mmol})$ was added to the solution of compound 25 (798 mg, $0.467 \mathrm{mmol})$ in dry $N, N$-dimethylmethanamide (DMF) $(15 \mathrm{~mL})$ at $0{ }^{\circ} \mathrm{C}$. After 30 min of stirring at room temperature, $\mathrm{MeI}(105 \mu \mathrm{L}, 1.68 \mathrm{mmol})$ was added to the reaction mixture and it was stirred for $4 \mathrm{~h}$. The reaction mixture was quenched by the addition of $\mathrm{MeOH}$ (4-5 drops). The solution was concentrated and the crude product was purified by gel chromatography (Sephadex LH-20 in $\mathrm{MeOH})$ to give $26(550 \mathrm{mg}, 67 \%)$ as a colourless syrup; $R_{\mathrm{f}}=0.58\left(9: 1 \mathrm{CH}_{2} \mathrm{Cl}_{2} / \mathrm{MeOH}\right) ;[\alpha]_{\mathrm{D}}+49.4$ (c 0.26, $\left.\mathrm{CHCl}_{3}\right) ;{ }^{1} \mathrm{H}-\mathrm{NMR}\left(\mathrm{CDCl}_{3}, 400 \mathrm{MHz}\right): \delta$ (ppm) 7.79-7.12 (m, 37H, arom.), $5.58(\mathrm{~d}, J=3.6 \mathrm{~Hz}$, $1 \mathrm{H}), 5.21(\mathrm{~d}, \mathrm{~J}=5.6 \mathrm{~Hz}, 1 \mathrm{H}), 5.00-4.33(\mathrm{~m}, 17 \mathrm{H}), 3.86-2.98\left(\mathrm{~m}, 55 \mathrm{H}, 20 \times\right.$ skeleton protons, $9 \times \mathrm{OCH}_{3}$, $\left.3 \times \mathrm{H6}_{\mathrm{a}, \mathrm{b}}, \mathrm{H}-7 \mathrm{a}, \mathrm{b}\right), 2.48-2.39\left(\mathrm{~m}, 1 \mathrm{H}, \mathrm{H}-6_{\mathrm{a}}-\mathrm{F}\right), 2.02-2.01\left(\mathrm{~m}, 1 \mathrm{H}, \mathrm{H}-6_{\mathrm{b}}-\mathrm{F}\right) ;{ }^{13} \mathrm{C}-\mathrm{NMR}\left(\mathrm{CDCl}_{3}, 100 \mathrm{MHz}\right): \delta$ (ppm) 169.8 (CO), 139.3, 138.9, 138.3, 138.2, 138.1, 138.0, 136.1, 133.2, $132.8\left(9 \times \mathrm{C}_{\mathrm{q}}\right.$ arom.), 128.3-125.6 (37C, arom.), 103.0, 100.1, 99.7, 98.0, 96.0 (5 × C-1), 86.3, 84.8, 83.3, 81.6, 80.7, 80.2, 79.8, 79.6, 79.5, 79.1, $78.3,76.4,74.3,74.2,72.0,71.5,70.7,70.2,69.9,69.6$ (20C, skeleton carbons), 75.2, 75.1, 74.6, 73.5, 73.3, 73.2, $72.9\left(7 \times \mathrm{ArCH}_{2}\right), 69.1,68.3,68.0(3 \times \mathrm{C}-6), 60.6,60.4,60.2,59.7,59.6,59.4,59.3\left(7 \times \mathrm{OCH}_{3}\right)$, $55.2\left(\mathrm{C}-1-\mathrm{OCH}_{3}\right), 51.7\left(\mathrm{COOCH}_{3}\right), 46.8$ (C-7-F), $26.4(\mathrm{C}-6-\mathrm{F}) ; \mathrm{MALDI}-\mathrm{TOF} \mathrm{MS}: \mathrm{m} / z 1771.76$ [M + Na] ${ }^{+}$ (Calcd. 1771.69). Anal. Calcd. for $\mathrm{C}_{93} \mathrm{H}_{113} \mathrm{NaO}_{29} \mathrm{~S}$ (1749.93): C, 63.83; H, 6.51; S, 1.83. Found: C, 63.92; H, 7.03; S, 2.01.

Methyl [6-O-benzyl-2,3,4-tri-O-methyl- $\alpha$-D-glucopyranosyl]-(1 $\rightarrow 4)-[2,3-d i-O-m e t h y l-\beta$-D-glucopyranosyl]$(1 \rightarrow 4)$-[sodium (2,3-di-O-benzyl-6-deoxy-6-C-sulfonatomethyl- $\alpha$-D-glucopyranosyl)]-(1 $\rightarrow 4)$-[methyl (2,3-di-Omethyl- $\alpha$-L-idopyranosyl)uronate]-( $\rightarrow 4)-(2,3,6-$ tri-O-benzyl- $\alpha$-D-glucopyranoside) (27). Compound 26 (550 $\mathrm{mg}, 0.314 \mathrm{mmol}$ ) was converted to 27 according to general method $\mathbf{D}$. The crude product was purified by column chromatography $\left(9: 1 \mathrm{CH}_{2} \mathrm{Cl}_{2} / \mathrm{MeOH}\right)$ to give $27(404 \mathrm{mg}, 80 \%)$ as a colourless syrup; $R_{\mathrm{f}}=0.52\left(9: 1 \mathrm{CH}_{2} \mathrm{Cl}_{2} / \mathrm{MeOH}\right) ;[\alpha]_{\mathrm{D}}+24.2\left(c 0.06, \mathrm{CHCl}_{3}\right) ;{ }^{1} \mathrm{H}-\mathrm{NMR}\left(\mathrm{CDCl}_{3}, 400 \mathrm{MHz}\right): \delta(\mathrm{ppm})$ 7.35-7.24 (m, 30H, arom.), 5.50 (s, 1H), $5.18(\mathrm{~s}, 2 \mathrm{H}), 4.82-4.57$ (m, 15H), 3.94-3.15 (m, 55H, 20 skeleton protons, $\left.9 \times \mathrm{OCH}_{3}, 3 \times \mathrm{H}-6_{\mathrm{a}, \mathrm{b}}, \mathrm{H}-7 \mathrm{a}, \mathrm{b}\right), 2.52-2.41\left(\mathrm{~m}, 1 \mathrm{H}, \mathrm{H}-6_{\mathrm{a}}-\mathrm{F}\right), 2.08-1.99\left(\mathrm{~m}, 1 \mathrm{H}, \mathrm{H}-6_{\mathrm{b}}-\mathrm{F}\right) ;{ }^{13} \mathrm{C}-\mathrm{NMR}$ $\left(\mathrm{CDCl}_{3}, 100 \mathrm{MHz}\right): \delta(\mathrm{ppm}) 171.7(\mathrm{CO}), 140.3-139.5$ (6 × $\mathrm{C}_{\mathrm{q}}$ arom. $), 129.5-128.4$ (30C, arom.), 103.5, 100.6, 99.0, 97.8, $97.0(5 \times \mathrm{C}-1), 87.6,86.4,84.4,83.0,81.7,81.4,80.9,80.6,80.5,80.4,79.7,76.8,76.6$ (20C, skeleton carbons), 76.6, 76.0, 74.9, 74.5, $74.1\left(6 \times \mathrm{ArCH}_{2}\right), 70.0,69.7,62.8(3 \times \mathrm{C}-6), 61.0,60.8,60.6,59.9$, $59.7\left(7 \times \mathrm{OCH}_{3}\right), 55.6\left(\mathrm{C}-1-\mathrm{OCH}_{3}\right), 52.6\left(\mathrm{COOCH}_{3}\right), 49.0(\mathrm{C}-7-\mathrm{F}), 27.8(\mathrm{C}-6-\mathrm{F}) ; \mathrm{MALDI}-\mathrm{TOF} \mathrm{MS}: \mathrm{m} / z$ 1631.77 [M + Na] ${ }^{+}$(Calcd. 1631.63). Anal. Calcd. for $\mathrm{C}_{82} \mathrm{H}_{105} \mathrm{NaO}_{29} \mathrm{~S}$ (1609.75): C, 61.18; H, 6.57; S, 1.99 . Found: C, 61.24; H, 6.59; S, 2.08. 
Methyl-(6-O-benzyl-2,3,4-tri-O-methyl- $\alpha$-D-glucopyranosyl)-(1 $\rightarrow 4)$-[sodium (2,3-di-O-methyl- $\beta$-D-glucopyranosyl) uronate]-(1 $\rightarrow 4)$-(sodium (2,3-di-O-benzyl-6-deoxy-6-C-sulfonatomethyl- $\alpha$-D-glucopyranosyl)-(1 $\rightarrow 4)$-[methyl (2,3-di-O-methyl- $\alpha$-L-idopyranosyl)uronate]-(1 $\rightarrow 4)-(2,3,6-t r i-O-b e n z y l-\alpha$-D-glucopyranoside) (28). To a vigorously stirred solution of $27(40 \mathrm{mg}, 0.025 \mathrm{mmol})$ in $\mathrm{CH}_{2} \mathrm{Cl}_{2}(2.0 \mathrm{~mL})$ and $\mathrm{H}_{2} \mathrm{O}(1.0 \mathrm{~mL}), \mathrm{TEMPO}$ ( $0.8 \mathrm{mg}, 0.005 \mathrm{mmol}, 0.2$ equiv.) and BAIB (32 mg, $0.099 \mathrm{mmol}, 4$ equiv.) were added. After $24 \mathrm{~h}$, the TLC (9:1 $\left.\mathrm{CH}_{2} \mathrm{Cl}_{2} / \mathrm{MeOH}\right)$ indicated moderate conversion of the starting material. Another portion of BAIB (32 mg, $0.099 \mathrm{mmol}, 4$ equiv.) were added and the stirring was continued for a further $24 \mathrm{~h}$. The reaction mixture was quenched by the addition of $10 \%$ aq $\mathrm{Na}_{2} \mathrm{~S}_{2} \mathrm{O}_{3}$ solution (4 mL). The mixture was then extracted twice with EtOAc $(8 \mathrm{~mL})$, and the combined organic layers were dried, and concentrated. The residue was purified by column chromatography $\left(9: 1 \mathrm{CH}_{2} \mathrm{Cl}_{2} / \mathrm{MeOH}\right)$ to give a colourless syrup (31 mg). The mass spectrum contained peaks corresponding to 28 and its partially debenzylated derivatives; this mixture was used in the subsequent reaction without further purification. MALDI-TOF MS for $\mathrm{C}_{82} \mathrm{H}_{102} \mathrm{Na}_{2} \mathrm{O}_{30} \mathrm{~S}$ (1644.60): $\mathrm{m} / z$ 1667.65 [M + Na] ${ }^{+}$(Calcd. 1667.59); $1379.56[\mathrm{M}+\mathrm{Na}(-3 \mathrm{Bn})]^{+}$(Calcd. 1379.45); $1487.56[\mathrm{M}+\mathrm{Na}(-2 \mathrm{Bn})]^{+}$(Calcd. 1487.49).

Nona sodium [methyl (2,3,4-tri-O-methyl-6-O-sulfonato- $\alpha$-D-glucopyranosyl)-(1 $\rightarrow 4)$-[sodium (2,3-di-Omethyl- $\beta$-D-glucopyranosyl)uronate]-(1 $\rightarrow 4)$-[sodium (2,3-di-O-sulfonato-6-deoxy-6-C-sulfonatomethyl- $\alpha$ D-glucopyranosyl)-(1 $\rightarrow 4$ )-[sodium (2,3-di-O-methyl- $\alpha$-L-idopyranosyl)uronate]-( $\rightarrow 4)-2,3,6$-tri-O-sulfonato- $\alpha$ D-glucopyranoside (29). An amount of $0.5 \mathrm{M} \mathrm{NaOH}$ solution $(0.30 \mathrm{~mL})$ was added to the solution of $28(30 \mathrm{mg})$ in a mixture of THF $(0.30 \mathrm{~mL})$ and $\mathrm{MeOH}(0.30 \mathrm{~mL})$ and stirred at room temperature for $24 \mathrm{~h}$. The reaction was quenched by the addition of $1 \mathrm{~N} \mathrm{HCl}$ solution (1 drop) and the mixture was concentrated. The crude product was converted to sodium salt by ion exchange resin (Dowex, $\mathrm{MeOH})$ to give a colourless syrup (28 mg). The syrupy residue was dissolved in $96 \% \mathrm{EtOH}(3.0 \mathrm{~mL})$ and $10 \%$ $\mathrm{Pd} / \mathrm{C}(10 \mathrm{mg})$ and acetic acid $(100 \mu \mathrm{L})$ were added. The mixture was stirred at room temperature for $24 \mathrm{~h}$ under a 10 bar $\mathrm{H}_{2}$ atmosphere. The mixture was diluted with $\mathrm{MeOH}$, the catalyst was filtered off through a pad of Celite and the filtrate was concentrated. The crude product was purified by column chromatography $\left(7: 6: 1 \mathrm{CH}_{2} \mathrm{Cl}_{2} / \mathrm{MeOH} / \mathrm{H}_{2} \mathrm{O}, R_{\mathrm{f}}=0.13\right)$ and gel chromatography (Sephadex G-25, $\left.\mathrm{H}_{2} \mathrm{O}\right)$ to give the corresponding pentaol $(9 \mathrm{mg})$ which was characterized by MALDI MS. (MALDI-TOF MS for $\mathrm{C}_{39} \mathrm{H}_{63} \mathrm{Na}_{3} \mathrm{O}_{30} \mathrm{~S}$ (1112.28): $m / z 1091.39$ [M $\left.-\mathrm{Na}+2 \mathrm{H}\right]^{+}$(Calcd. 1091.31), $1113.39[\mathrm{M}+\mathrm{H}]^{+}$ (Calcd. 1113.29), $1135.42[\mathrm{M}+\mathrm{Na}]^{+}$(Calcd. 1135.27). To the solution of the pentaol derivative $(9 \mathrm{mg})$ in dry DMF $(0.6 \mathrm{~mL}), \mathrm{SO}_{3} \cdot \mathrm{Et}_{3} \mathrm{~N}$ complex $(44 \mathrm{mg}, 0.040 \mathrm{mmol})$ was added and the reaction mixture was stirred at $50{ }^{\circ} \mathrm{C}$ for $48 \mathrm{~h}$. The reaction was quenched with satd. aq. $\mathrm{NaHCO}_{3}(21 \mathrm{mg}, 0.24 \mathrm{mmol})$. The solution was concentrated. The crude product was treated with Dowex ion-exchange resin $\left(\mathrm{Na}^{+}\right.$form), and then purified by Sephadex G-25 column chromatography eluting with $\mathrm{H}_{2} \mathrm{O}$ to give 29 (7 mg, 17\% from 27) as a white solid. ESI-MS for $\mathrm{C}_{39} \mathrm{H}_{57} \mathrm{Na}_{9} \mathrm{O}_{48} \mathrm{~S}_{7}(1723.91): m / z 795.110[\mathrm{M}-6 \mathrm{Na}+4 \mathrm{H}]^{2-}$ (Calcd. 795.00); $522.451[\mathrm{M}-7 \mathrm{Na}+4 \mathrm{H}]^{3-}$ (Calcd.: 522.34).

Methyl [2,3,4-tri-O-methyl-6-deoxy-6-C-(ethylsulfonatomethyl)- $\alpha$-D-glucopyranosyl]-(1 $\rightarrow 4)-[2,3-$ di-O-acetyl$\beta$-D-glucopyranosyl]-(1 $\rightarrow 4)$-[2,3-di-O-benzyl-6-deoxy-6-C-(ethylsulfonatomethyl)- $\alpha$-D-glucopyranosyl]-(1 $\rightarrow 4)$ [methyl (2-O-acetyl-3-O-methyl- $\alpha$-L-idopyranosyl)uronate]-(1 $\rightarrow 4)-(2,3,6-$ tri-O-benzyl- $\alpha$-D-glucopyranoside) (30). Compound $6(975 \mathrm{mg}, 0.53 \mathrm{mmol})$ was converted to 30 according to general method D. The crude product was purified by column chromatography ( $94: 6 \mathrm{CH}_{2} \mathrm{Cl}_{2}$ /acetone) to give compound $30(612 \mathrm{mg}, 68 \%)$ as a colourless syrup; $R_{\mathrm{f}}=0.26\left(93: 7 \mathrm{CH}_{2} \mathrm{Cl}_{2}\right.$ /acetone $) ;[\alpha]_{\mathrm{D}}+30.56\left(c 0.10, \mathrm{CHCl}_{3}\right)$; ${ }^{1} \mathrm{H}-\mathrm{NMR}\left(360 \mathrm{MHz}, \mathrm{CDCl}_{3}\right.$ ) $\delta 7.45-7.17$ (m, 25H, arom.), 5.23 (t, J = 9.3 Hz, 1H, H-3-E), 5.11 (s, $1 \mathrm{H}$, H-1-G), 5.00 (d, J = 3.6 Hz, 1H, H-1-D), 4.96-4.49 (m, 16H, H-2-E, H-5-G, H-2-G, H-1-E, H-1-H, H-1-F, $\left.10 \times \mathrm{PhCH}_{2}\right), 4.29\left(\mathrm{q}, J=7.1,1.2 \mathrm{~Hz}, 4 \mathrm{H}, 2 \times \mathrm{SO}_{3} \mathrm{CH}_{2} \mathrm{CH}_{3}\right), 3.93-3.07(\mathrm{~m}, 21 \mathrm{H}$, skeleton protons), 3.55, 3.52, 3.41, 3.41, 3.34, $3.31\left(6 \mathrm{~s}, 18 \mathrm{H}, 6 \times \mathrm{OCH}_{3}\right), 3.01(\mathrm{dd}, J=9.8,3.6 \mathrm{~Hz}, 1 \mathrm{H}, \mathrm{H}-2-\mathrm{D}), 2.72(\mathrm{t}, J=9.3 \mathrm{~Hz}$, 1H, H-4-D), 2.34-2.22 (m, 2H, H-6a $\left.-\mathrm{D}, \mathrm{H}-6_{\mathrm{a}}-\mathrm{F}\right), 2.03,2.01,1.96$ (3s, 9H, $\left.3 \times \mathrm{COCH}_{3}\right), 1.94-1.75(\mathrm{~m}, 3 \mathrm{H}$, H-6 $\left.-\mathrm{b}, \mathrm{H}-6_{\mathrm{b}}-\mathrm{F}, \mathrm{OH}\right), 1.41\left(\mathrm{~m}, 6 \mathrm{H}, 2 \times \mathrm{SO}_{3} \mathrm{CH}_{2} \mathrm{CH}_{3}\right) \mathrm{ppm} ;{ }^{13} \mathrm{C}-\mathrm{NMR}\left(91 \mathrm{MHz}, \mathrm{CDCl}_{3}\right) \delta 170.2,170.0$, $169.6,169.6(4 \times \mathrm{CO}), 139.1,139.0,138.1,138.1,137.9\left(5 \mathrm{C}, \mathrm{C}_{\mathrm{q}}\right.$ arom. $), 128.6,128.6,128.4,128.4,128.2$, $128.1,127.9,127.8,127.6,127.3,127.1,126.1$ (25C, arom.), 100.9, 98.4, 98.0, 97.6, 96.8 (5 × C-1), 83.8, 82.8, 
82.1, 81.7, 80.2, 79.8, 79.4, 79.1, 76.1, 75.3, 74.8, 74.2, 72.6, 72.2, 70.2, 69.6, 69.2, 68.4, 67.5 (20C, skeleton carbons), 74.6, 73.9, 73.4, 73.4, $73.4\left(5 \times \mathrm{PhCH}_{2}\right), 68.4,60.6(\mathrm{C}-6-\mathrm{H}, \mathrm{C}-6-\mathrm{E}), 66.3,66.1\left(2 \times \mathrm{SO}_{3} \mathrm{CH}_{2} \mathrm{CH}_{3}\right)$, 60.8, 60.7, 59.5, 58.2, 55.3, $51.8\left(6 \times \mathrm{OCH}_{3}\right), 46.8,46.6$ (C-7-D, C-7-F), 26.4, 25.5 (C-6-D, C-6-F), 21.1, 21.0, $20.6\left(3 \times \mathrm{COCH}_{3}\right), 15.2,15.2\left(2 \times \mathrm{SO}_{3} \mathrm{CH}_{2} \mathrm{CH}_{3}\right)$ ppm; MALDI-TOF MS: $m / z 1737.62[\mathrm{M}+\mathrm{Na}]^{+}(\mathrm{Calcd}$. 1737.62); Anal. Calcd. for $\mathrm{C}_{83} \mathrm{H}_{110} \mathrm{O}_{34} \mathrm{~S}_{2}$ (1714.63): C, 58.10; H, 6.46; O, 31.70; S, 3.74. Found: C, 58.21; $\mathrm{H}, 6.41 ; \mathrm{S}, 3.70$.

Methyl [2,3,4-tri-O-methyl-6-deoxy-6-C-(ethylsulfonatomethyl)- $\alpha$-D-glucopyranosyl]-(1 $\rightarrow 4)$-[sodium (2,3-di-O-acetyl- $\beta$-D-glucopyranosyl)uronate]-(1 $\rightarrow 4)$-[2,3-di-O-benzyl-6-deoxy-6-C-(ethylsulfonatomethyl)- $\alpha$ D-glucopyranosyl]-(1 $\rightarrow 4$ )-[methyl (2-O-acetyl-3-O-methyl- $\alpha$-L-idopyranosyl) uronate]-(1 $\rightarrow 4)-(2,3,6$-tri-Obenzyl- $\alpha$-D-glucopyranoside) (31). Compound 30 (2.4 g, $1.87 \mathrm{mmol})$ was converted to 31 according to general method $\mathbf{A}$. The reaction mixture was stirred for $24 \mathrm{~h}$. The crude product was purified by column chromatography $\left(98: 2 \mathrm{CH}_{2} \mathrm{Cl}_{2} / \mathrm{MeOH}\right)$ to give $31(528 \mathrm{mg}, 80 \%)$ as a colourless syrup; $R_{\mathrm{f}}=0.50\left(95: 5 \mathrm{CH}_{2} \mathrm{Cl}_{2} / \mathrm{MeOH}\right) ;[\alpha]_{\mathrm{D}}+29.26\left(c\right.$ 0.11, $\left.\mathrm{CHCl}_{3}\right) ;{ }^{1} \mathrm{H}-\mathrm{NMR}\left(360 \mathrm{MHz}, \mathrm{CDCl}_{3}\right) \delta 7.38-7.19$ (m, 25H, arom.), 5.26-5.18 (m, 1H), 5.08 (s, 1H, H-1-G), 5.00 (d, J = 3.5 Hz, 1H, H-1-D), 4.96-4.49 (m, $16 \mathrm{H}, 10 \times \mathrm{PhCH}_{2}$, skeleton protons), 4.30, $4.28\left(2 \mathrm{q}, 4 \mathrm{H}, 2 \times \mathrm{SO}_{3} \mathrm{CH}_{2} \mathrm{CH}_{3}\right), 4.04(\mathrm{t}, J=8.8 \mathrm{~Hz}, 1 \mathrm{H})$, 3.93-3.77 ( $\mathrm{m}, 4 \mathrm{H}$, skeleton protons), 3.77-3.60 ( $\mathrm{m}, 4 \mathrm{H}$, skeleton protons), 3.59-3.18 ( $\mathrm{m}, 8 \mathrm{H}$ skeleton protons), 3.56, 3.52, 3.42, 3.39, 3.34, $3.30\left(6 \mathrm{~s}, 18 \mathrm{H}, 6 \times \mathrm{OCH}_{3}\right), 3.16-3.05(\mathrm{~m}, 1 \mathrm{H}, \mathrm{H}-7 \mathrm{~b}), 3.01(\mathrm{dd}, J=9.8$, 3.6 Hz, 1H, H-2-D), 2.72 (t, J = 9.3 Hz, 1H, H-4-D), 2.33-2.17 (m, 2H, H-6a-D, H-6a-F), 2.03, $2.01,1.95$ $\left(3 \mathrm{~s}, 9 \mathrm{H}, 3 \times \mathrm{COCH}_{3}\right), 1.91-1.77\left(\mathrm{~m}, 2 \mathrm{H}, \mathrm{H}-6_{\mathrm{b}}-\mathrm{D}, \mathrm{H}-6_{\mathrm{b}}-\mathrm{F}\right), 1.39,1.38\left(\mathrm{~m}, 6 \mathrm{H}, 2 \times \mathrm{SO}_{3} \mathrm{CH}_{2} \mathrm{CH}_{3}\right) \mathrm{ppm}$; ${ }^{13} \mathrm{C}-\mathrm{NMR}\left(91 \mathrm{MHz}, \mathrm{CDCl}_{3}\right) \delta 170.4,169.9,169.5,169.5,169.0(5 \times \mathrm{CO}), 139.1,138.7,138.1,138.1,137.8$ (5C, $C_{\mathrm{q}}$ arom.), 128.6, 128.5, 128.4, 128.2, 128.2, 128.1, 127.9, 127.8, 127.7, 127.6, 127.5, 127.3, 127.2 (25C, arom.), 100.8, 98.4, 98.0, 97.6, 97.6 (5 × C-1), 83.8, 82.7, 81.9, 81.6, 80.2, 80.1, 79.8, 79.0, 76.2, $75.3,74.7,74.4,74.4,74.0,72.4,70.2,69.5,69.1,68.3,67.4$ (20C, skeleton carbons), 74.9, 74.8, 73.8, 73.4, $73.4\left(5 \times \mathrm{PhCH}_{2}\right), 68.5(\mathrm{C}-6-\mathrm{H}), 66.6,66.4\left(2 \times \mathrm{SO}_{3} \mathrm{CH}_{2} \mathrm{CH}_{3}\right), 60.7,60.7,59.5,58.2,55.3,51.9$ $\left(6 \times \mathrm{OCH}_{3}\right), 46.6,46.5(\mathrm{C}-7-\mathrm{D}, \mathrm{C}-7-\mathrm{F}), 26.0,25.7$ (C-6-D, C-6-F), 21.1, 20.9, $20.6\left(3 \times \mathrm{COCH}_{3}\right), 15.2,15.2$ $\left(2 \times \mathrm{SO}_{3} \mathrm{CH}_{2} \mathrm{CH}_{3}\right)$ ppm; MALDI-TOF MS: $m / z$ 1773.53 [M + Na] ${ }^{+}$(Calcd. 1773.58); Anal. Calcd. for $\mathrm{C}_{83} \mathrm{H}_{107} \mathrm{O}_{35} \mathrm{~S}_{2}$ (1714.63): C, 58.10; H, 6.46; O, 31.70; S, 3.74. Found: C, 58.21; H, 6.41; S, 3.70.

Methyl [2,3,4-tri-O-methyl-6-deoxy-6-C-(sulfonatomethyl)- $\alpha$-D-glucopyranosyl]-(1 $\rightarrow 4)$-[sodium $(\beta$-D-glucopyranosyl)uronate]-(1 $\rightarrow 4)$-[2,3-di-O-benzyl-6-deoxy-6-C-(sulfonatomethyl)- $\alpha$-D-glucopyranosyl]$(1 \rightarrow 4)$-[methyl (3-O-methyl- $\alpha$-L-idopyranosyl)uronate]-(1 $\rightarrow 4)-(2,3,6$-tri-O-benzyl- $\alpha$-D-glucopyranoside) (32). $\mathrm{NaOMe}(2 \mathrm{mg}, 0.03 \mathrm{mmol})$ was added to the solution of compound 31 (500 $\mathrm{mg}, 0.29 \mathrm{mmol}$ ) and stirred at room temperature for $24 \mathrm{~h}$. The mixture was quenched by the addition of acetic acid (1-2 drops) and then concentrated. The crude product was dissolved in acetone $(20 \mathrm{~mL})$ and NaI $(128 \mathrm{mg}, 0.86 \mathrm{mmol})$ was added to the solution. The reaction mixture was stirred at room temperature for $24 \mathrm{~h}$. The mixture was concentrated and the crude product was purified by gel chromatography (Sephadex LH-20, $\mathrm{MeOH}$ ) to give 32 (386 mg, 84\% for two steps) as a colourless syrup. $R_{\mathrm{f}}=0.47\left(7: 3 \mathrm{CH}_{2} \mathrm{Cl}_{2} / \mathrm{MeOH}\right)$; $[\alpha]_{\mathrm{D}}+34.32\left(c\right.$ 1.66, $\left.\mathrm{CHCl}_{3}\right) ;{ }^{1} \mathrm{H}-\mathrm{NMR}\left(360 \mathrm{MHz}, \mathrm{CDCl}_{3}\right) \delta 7.44-7.15$ (m, 25H, arom.), $5.51(\mathrm{~d}, \mathrm{~J}=3.5 \mathrm{~Hz}$, $1 \mathrm{H}), 5.10(\mathrm{~s}, 1 \mathrm{H}), 5.03(\mathrm{~d}, J=3.3 \mathrm{~Hz}, 1 \mathrm{H}), 5.02-4.50\left(\mathrm{~m}, 14 \mathrm{H}, 5 \times \mathrm{PhCH}_{2}\right.$, skeleton protons $), 3.99-3.21$ (m, 22H, skeleton protons), 3.57, 3.52, 3.52, 3.46, 3.36, $3.35\left(6 \mathrm{~s}, 18 \mathrm{H}, 6 \times \mathrm{OCH}_{3}\right), 3.13(\mathrm{dd}, \mathrm{J}=9.8,3.6$ Hz, 1H, H-2-D), 3.08-2.95 (m, 2H, H-7a-D, H-7a-F), 2.84-2.75 (m, 1H, H-4-D), 2.43-2.31 (m, 1H, H-6a), 2.30-2.18 (m, 1H, H-6 $\left.{ }_{\mathrm{a}}\right), 2.11-1.78\left(\mathrm{~m}, 2 \mathrm{H}, 2 \times \mathrm{H}-6_{\mathrm{b}}\right)$ ppm; ${ }^{13} \mathrm{C}-\mathrm{NMR}\left(91 \mathrm{MHz}, \mathrm{CDCl}_{3}\right) \delta 177.8,171.6$ $(2 \times \mathrm{CO}), 140.2,140.1,139.5,139.4,139.0$ (5C, $C_{\mathrm{q}}$ arom.), 129.6, 129.3, 129.2, 129.1, 128.9, 128.7, 128.4 (25C, arom.), 104.3, 102.3, 98.9, 98.2, 95.9 (5 × C-1), 85.1, 83.7, 81.8, 81.2, 80.8, 80.4, 79.3, 78.4, 78.2, 76.2, $75.9,75.7,75.2,73.6,72.5,71.7,70.9,70.7,69.1,67.8$ (20C, skeleton carbons), 76.3, 76.1, 75.0, 74.5, 74.0 $\left(5 \times \mathrm{PhCH}_{2}\right), 69.8(\mathrm{C}-6-\mathrm{H}), 60.8,60.6,59.3,59.2,58.5,55.5\left(6 \times \mathrm{OCH}_{3}\right), 28.3,27.8(\mathrm{C}-6-\mathrm{D}, \mathrm{C}-6-\mathrm{F}) \mathrm{ppm}$; MALDI-TOF MS: $m / z 1635.50[\mathrm{M}+\mathrm{Na}]^{+}$(Calcd. 1635.45); Anal. Calcd. for $\mathrm{C}_{73} \mathrm{H}_{91} \mathrm{Na}_{3} \mathrm{O}_{32} \mathrm{~S}_{2}(1612.46)$ : C, 54.34; H, 5.68; Na, 4.27; O, 31.73; S, 3.97. Found: C, 54.23; H, 5.66; S, 4.03. 
Methyl-[sodium 2,3,4-tri-O-methyl-6-deoxy-6-C-(sulfonatomethyl)- $\alpha$-D-glucopyranosyl]-(1 $\rightarrow 4$ )-[sodium (2,3-di-O-methyl- $\beta$-D-glucopyranosyl)uronate]-(1 $\rightarrow 4)$-[2,3-di-O-benzyl-6-deoxy-6-C-(sulfonatomethyl)- $\alpha$ - $D$ glucopyranosyl]-(1 $\rightarrow 4)$-[methyl (2,3-di-O-methyl- $\alpha$-L-idopyranosyl)uronate]-( $\rightarrow 4$-2,3,6-tri-O-benzyl- $\alpha$ - $D$ glucopyranoside (33). An amount of $60 \mathrm{~m} / \mathrm{m} \% \mathrm{NaH}(55 \mathrm{mg}, 1.38 \mathrm{mmol})$ was added to the solution of compound $32(370 \mathrm{mg}, 0.23 \mathrm{mmol})$ in dry DMF $(40 \mathrm{~mL})$ at $0{ }^{\circ} \mathrm{C}$. After $30 \mathrm{~min}$ of stirring at room temperature, MeI $(64 \mu \mathrm{L}, 1.04 \mathrm{mmol})$ was added to the reaction mixture and it was stirred for $4 \mathrm{~h}$. The reaction mixture was quenched by the addition of $\mathrm{MeOH}$ and acetic acid (1-2 drops). The solution was concentrated and the crude product was purified by gel chromatography (Sephadex LH-20, MeOH) to give $33(249 \mathrm{mg}, 65 \%)$ as a colourless syrup; $R_{\mathrm{f}}=0.53\left(7: 3 \mathrm{CH}_{2} \mathrm{Cl}_{2} / \mathrm{MeOH}\right) ;[\alpha]_{\mathrm{D}}+4.09\left(c 0.81, \mathrm{CHCl}_{3}\right)$; ${ }^{1} \mathrm{H}-\mathrm{NMR}\left(360 \mathrm{MHz}, \mathrm{CDCl}_{3}\right) \delta 7.49-7.10$ (m, 25H, arom.), 5.15-4.51 (m, 17H, $5 \times \mathrm{H}-1, \mathrm{H}-5-\mathrm{E}, \mathrm{H}-5-\mathrm{G}$, $\left.10 \times \mathrm{PhCH}_{2}\right), 4.00-3.24$ (m, 20H, skeleton protons), 3.57, 3.55, 3.53, 3.49, 3.46, 3.43, 3.38, 3.36, 3.35 (9s, $\left.27 \mathrm{H}, 9 \times \mathrm{OCH}_{3}\right), 3.11(\mathrm{dd}, J=9.7,3.6 \mathrm{~Hz}, 1 \mathrm{H}, \mathrm{H}-2-\mathrm{D}), 3.09-2.77(\mathrm{~m}, 2 \mathrm{H}, 2 \times \mathrm{H}-7 \mathrm{~b}), 2.56-2.38(\mathrm{~m}, 1 \mathrm{H}$, H-6a $), 2.30-2.17$ (m, 1H, H-6a ), 2.07-1.85 (m, 2H, $2 \times$ H-6 $)$ ppm; ${ }^{13}$ C-NMR (101 MHz, MeOD) $\delta 170.4$, $170.0(2 \times \mathrm{CO}), 139.3,139.3,138.5,138.4,138.4$ (5C, $C_{\mathrm{q}}$ arom.), 128.8, 128.7, 128.6, 128.5, 128.3, 128.3, 128.2, 128.1, 128.0, 127.9, 127.6 (25C, arom.), 100.1, 100.0, 98.4, 96.7, 96.0 (5 × C-1), 86.2, 84.2, 83.8, 83.5, 82.7, 82.1, 81.7, 81.4, 80.3, 79.5, 79.2, 78.9, 76.5, 74.7, 74.3, 71.6, 71.4, 70.7, 70.1, 68.8 (20C, skeleton carbons), 75.7, 75.5, 75.2,73.8, $73.8\left(5 \times \mathrm{PhCH}_{2}\right), 73.9(\mathrm{C}-6-\mathrm{H}), 60.9,60.9,60.1,60.0,59.8,59.3,55.5,53.0$, 52.3, $\left(9 \times \mathrm{COCH}_{3}\right), 47.6,47.5(\mathrm{C}-7-\mathrm{D}, \mathrm{C}-7-\mathrm{F}), 27.5,27.3$ (C-6-D, C-6-F) ppm; MALDI-TOF MS: $m / z$ $1655.53[\mathrm{M}+\mathrm{Na}]^{+}$(Calcd. 1655.52); Anal. Calcd. for $\mathrm{C}_{76} \mathrm{H}_{97} \mathrm{Na}_{3} \mathrm{O}_{32} \mathrm{~S}_{2}$ (1654.51): C, 55.13; $\mathrm{H}, 5.91 ; \mathrm{Na}$, 4.17; O, 30.92; S, 3.87. Found: C, 55.20; H, 5.97; S, 3.79.

Methyl [2,3,4-tri-O-methyl-6-deoxy-6-C-sulfonatomethyl- $\alpha$-D-glucopyranosyl]-(1 $\rightarrow 4)$-[sodium $\quad(2,3-d i-O-$ methyl- $\beta$-D-glucopyranosyl)uronate]-(1 $\rightarrow 4)$-[2,3-di-O-benzyl-6-deoxy-6-C-(sulfonatomethyl)- $\alpha$-D-glucopyranosyl]$(1 \rightarrow 4)$-[sodium (2,3-di-O-methyl- $\alpha$-L-idopyranosyl)uronate]-( $\rightarrow 4)-(2,3,6$-tri-O-benzyl- $\alpha$-D-glucopyranoside) (34). An amount of $0.5 \mathrm{M} \mathrm{NaOH}$ solution (2 mL) was added to the solution of compound 33 (206 mg, $0.12 \mathrm{mmol})$ in a mixture of THF $(2 \mathrm{~mL})$ and $\mathrm{MeOH}(2 \mathrm{~mL})$ and stirred at room temperature for $24 \mathrm{~h}$. The reaction was quenched by the addition of $1 \mathrm{~N} \mathrm{HCl}$ solution (1-2 drops) and the mixture was concentrated. The crude product was converted to sodium salt by ion exchange resin (Dowex, $\mathrm{MeOH})$ to give $34(187 \mathrm{mg}, 90 \%)$ as a colourless syrup; $R_{\mathrm{f}}=0.24\left(8: 2 \mathrm{CH}_{2} \mathrm{Cl}_{2} / \mathrm{MeOH}\right) ;[\alpha]_{\mathrm{D}}+42.81$ (c $\left.0.10, \mathrm{CHCl}_{3}\right) ;{ }^{1} \mathrm{H}-\mathrm{NMR}\left(360 \mathrm{MHz}, \mathrm{CDCl}_{3}\right) \delta 7.43-7.20(\mathrm{~m}, 25 \mathrm{H}$, arom.), 5.46 (d, J = 3.6 Hz, $1 \mathrm{H}), 5.15$ $(\mathrm{d}, J=3.3 \mathrm{~Hz}, 1 \mathrm{H}), 5.10(\mathrm{~d}, J=3.9 \mathrm{~Hz}, 1 \mathrm{H}), 5.04-4.48\left(\mathrm{~m}, 14 \mathrm{H}, 2 \times \mathrm{H}-1, \mathrm{H}-5-\mathrm{E}, \mathrm{H}-5-\mathrm{G}, 10 \times \mathrm{PhCH}_{2}\right)$, 4.09-3.25 (m, 17H, skeleton protons), 3.58, 3.54, 3.53, 3.52, 3.50, 3.43, 3.35, $3.34\left(8 \mathrm{~s}, 24 \mathrm{H}, 8 \times \mathrm{OCH}_{3}\right)$, 3.23-3.16 (m, 1H), 3.09 (dd, 1H, H-2-D), 3.07-3.86 (m, 2H, H-7b-D, H-7b-F), $2.81(\mathrm{t}, J=9.8 \mathrm{~Hz}, 1 \mathrm{H}$, H-4-D), 2.60-2.48 (m, 1H, H-6a ), 2.33-2.19 (m, 1H, H-6a), 1.98-1.82 (m, 2H, H-6 - -D, H-6 $-\mathrm{b})$ ppm; ${ }^{13}$ C-NMR (101 MHz, MeOD) $\delta 171.1,170.7$ (2× CO), 140.4, 140.2, 139.6, 139.5, 139.5 (5C, $C_{\mathrm{q}}$ arom.), $129.4,129.3,129.2,129.0,129.0,128.8,128.7,128.6,128.4,128.2,128.0$ (25C, arom.), 104.6, 100.5, 98.9, 96.8, $96.7(5 \times C-1), 87.0,85.4,84.5,84.1,82.9,82.8,81.3,81.2,80.8,80.6,80.4,76.6,75.2,74.9,74.1$, 71.6, 71.5, 71.0, 70.7 (20C, skeleton carbons), 76.0, 75.8, 74.5, 73.9, $73.8\left(5 \times \mathrm{PhCH}_{2}\right), 69.6(\mathrm{C}-6-\mathrm{H}), 61.2$, $60.9,60.6,59.9,59.5,55.9,55.9,55.6\left(8 \times \mathrm{OCH}_{3}\right), 30.7,28.3(\mathrm{C}-6-\mathrm{D}, \mathrm{C}-6-\mathrm{F}) \mathrm{ppm}$; MALDI-TOF MS: $m / z$ $1685.49[\mathrm{M}+\mathrm{Na}]^{+}$(Calcd. 1685.47); Anal. Calcd. for $\mathrm{C}_{75} \mathrm{H}_{94} \mathrm{Na}_{4} \mathrm{O}_{32} \mathrm{~S}_{2}$ (1662.48): C, 54.15; $\mathrm{H}, 5.70 ; \mathrm{Na}$, 5.53; O, 30.78; S, 3.85. Found: C, 54.08; H, 5.67; S, 3.87.

Methyl [2,3,4-tri-O-methyl-6-deoxy-6-C-sulfonatomethyl- $\alpha$-D-glucopyranosyl]-(1 $\rightarrow$ 4)-[sodium (2,3-di-O-methyl$\beta$-D-glucopyranosyl)uronate]-(1 $\rightarrow 4)$-[6-deoxy-6-C-sulfonatomethyl- $\alpha$-D-glucopyranosyl]-(1 $\rightarrow 4)$-[sodium (2,3-di-O-methyl- $\alpha$-L-idopyranosyl)uronate]-(1 $\rightarrow 4)$ - $\alpha$-D-glucopyranoside (35). An amount of $10 \% \mathrm{Pd} / \mathrm{C}$ $(110 \mathrm{mg})$ and acetic acid $(350 \mu \mathrm{L})$ were added to the solution of compound $34(180 \mathrm{mg}, 0.11 \mathrm{mmol})$ in $96 \mathrm{v} / \mathrm{v} \% \mathrm{EtOH}(10 \mathrm{~mL})$. The mixture was stirred at room temperature for $24 \mathrm{~h}$ under a $10 \mathrm{bar}_{2}$ atmosphere. The mixture was diluted with $\mathrm{MeOH}$ and the catalyst was filtered off on Celite-pad. The filtrate was concentrated. The crude product was purified by column chromatography (7:6:1 $\mathrm{CH}_{2} \mathrm{Cl}_{2} / \mathrm{MeOH} / \mathrm{H}_{2} \mathrm{O}$ ) and gel chromatography (Sephadex $\left.\mathrm{G}-25, \mathrm{H}_{2} \mathrm{O}\right)$ to give $35(123 \mathrm{mg}, 92 \%)$ as a colourless syrup; $R_{\mathrm{f}}=0.25\left(7: 6: 1 \mathrm{CH}_{2} \mathrm{Cl}_{2} / \mathrm{MeOH} / \mathrm{H}_{2} \mathrm{O}\right) ;[\alpha]_{\mathrm{D}}+21.82$ (c $\left.0.21, \mathrm{CHCl}_{3}\right) ;{ }^{1} \mathrm{H}-\mathrm{NMR}$ 
$\left(360 \mathrm{MHz}, \mathrm{CDCl}_{3}\right) \delta 5.54(\mathrm{~s}, 1 \mathrm{H}), 5.14(\mathrm{~s}, 1 \mathrm{H}), 5.09(\mathrm{~s}, 1 \mathrm{H}), 4.83(\mathrm{~s}, 2 \mathrm{H}), 4.62(\mathrm{~s}, 1 \mathrm{H}), 4.18(\mathrm{~s}, 1 \mathrm{H}), 3.97-3.25$ $\left(\mathrm{m}, 46 \mathrm{H}\right.$, skeleton protons, $\left.8 \times \mathrm{OCH}_{3}\right), 3.18-2.96(\mathrm{~m}, 5 \mathrm{H}), 2.90(\mathrm{~d}, J=2.7 \mathrm{~Hz}, 1 \mathrm{H}), 2.49-2.35(\mathrm{~m}, 1 \mathrm{H}$, H-6a ), 2.29-2.16 (m, 1H, H-6a ), 1.99-1.85 (m, 2H, H-6, H-6 $)$ ppm; ${ }^{13}$ C-NMR (91 MHz, CDCl 3 ) $\delta 193.3$, $193.2(2 \times \mathrm{CO}), 103.5,103.4,100.1,100.0,96.2$ (5 × C-1), 86.8, 84.4, 83.9, 83.5, 82.3, 81.8, 80.4, 78.6, 78.1, $76.7,73.9,72.8,72.6,72.5,72.4,71.9,71.6,71.4,70.3,69.9$ (20C, skeleton carbons), 61.2 (C-6-H), 61.5, 61.1, 60.4, 60.4, 59.9, 59.6, 58.9, $55.9\left(8 \times \mathrm{OCH}_{3}\right), 48.3$, $48.2(\mathrm{C}-7-\mathrm{D}, \mathrm{C}-7-\mathrm{F}), 27.3,27.0(\mathrm{C}-6-\mathrm{D}, \mathrm{C}-6-\mathrm{F}) \mathrm{ppm}$; ESI-MS: $m / z 561.46[\mathrm{M}+2 \mathrm{H}]^{2-}$ (Calcd. 561.15); Anal. Calcd. for $\mathrm{C}_{40} \mathrm{H}_{64} \mathrm{Na}_{4} \mathrm{O}_{32} \mathrm{~S}_{2}(1212.24): \mathrm{C}, 39.61$; $\mathrm{H}, 5.32 ; \mathrm{Na}, 7.58 ; \mathrm{O}, 42.21 ; \mathrm{S}, 5.29$. Found: C, 39.56; H, 5.29; S, 5.24.

Nona sodium [methyl (2,3,4-tri-O-methyl-6-deoxy-6-C-sulfonatomethyl- $\alpha$-D-glucopyranosyl)]-(1 $\rightarrow 4)-[2,3-d i-O-$ methyl- $\beta$-D-glucopyranosyluronate]-(1 $\rightarrow 4)$-[2,3-di-O-sulfonato-6-deoxy-6-C-sulfonatomethyl- $\alpha$-D-glucopyranosyl]$(1 \rightarrow 4)$-[2,3-di-O-methyl- $\alpha$-L-idopyranosyluronate]-(1 $\rightarrow 4)-2,3,6$-tri-O-sulfonato- $\alpha$ - $D$-glucopyranoside (36). To the solution of compound $35(58 \mathrm{mg}, 0.047 \mathrm{mmol})$ in dry DMF (4 mL) sulfur trioxide-triethylamine complex $(215 \mathrm{mg}, 1.187 \mathrm{mmol})$ was added and the reaction mixture was stirred at $50{ }^{\circ} \mathrm{C}$ for $72 \mathrm{~h}$. The reaction was quenched with satd. aq. $\mathrm{NaHCO}_{3}(262 \mathrm{mg}, 3.12 \mathrm{mmol})$. The solution was concentrated. The crude product was treated with Dowex ion-exchange resin $\left(\mathrm{Na}^{+}\right.$form), and then purified by Sephadex G-25 column chromatography eluting with $\mathrm{H}_{2} \mathrm{O}$ to give $36(28 \mathrm{mg}, 36 \%)$ as a white powder. $R_{\mathrm{f}}=0.53\left(7: 4: 1 \mathrm{CH}_{2} \mathrm{Cl}_{2} / \mathrm{MeOH} / \mathrm{H}_{2} \mathrm{O}\right)$; ESI-MS: $m / z$ for $\mathrm{C}_{40} \mathrm{H}_{59} \mathrm{Na}_{9} \mathrm{O}_{47} \mathrm{~S}_{7}(1721.94): 837.771$ $[\mathrm{M}-2 \mathrm{Na}]^{2-}$ (Calcd. 837.979); 794.136 [M - 6Na + 4H $]^{2-}$ (Calcd. 794.015).

\section{Conclusions}

Five new idraparinux-analogue pentasaccharide precursors bearing one or two primary sulfonatomethyl moieties at the $\mathbf{D}, \mathbf{F}$ or $\mathbf{H}$ glucose units have been prepared using four disaccharides and two monosaccharides as the building blocks. The synthetic approach, including two subsequent glycosylation steps proved to be highly efficient, and the glycosylation reactions proceeded in good to excellent yields with complete stereoselectivity, regardless of the $C$-sulfonation pattern of the building blocks.

Unexpectedly, the transformation of the protected pentasaccharides into the fully $\mathrm{O}$-sulfated and $O$-methylated end-products was troublesome. Upon synthesis of pentasaccharide 29 from 9 via a Zemplén deacetylation, O-methylation, NAP-deprotection and TEMPO-BAIB oxidation route, the glucuronide formation proceeded with low efficacy. Fortunately, pentasaccharide 6 could be converted into the desired disulfonic acid product in an acceptable yield by applying a reaction sequence in which the oxidative formation of the glucuronic acid residue preceded the introduction of the methyl ether groups. A study to improve the yields of the synthetic procedures at a pentasaccharide level is in progress in our laboratory.

Synthesis of further isosteric sulfonic acid analogues of idraparinux and evaluation of their anticoagulant activity will be reported in due course.

Supplementary Materials: Supplementary materials can be accessed at: http:/ /www.mdpi.com/1420-3049/21/ 11/1497/s1. copies of ${ }^{1} \mathrm{H}-\mathrm{NMR}$ and ${ }^{13} \mathrm{C}-\mathrm{NMR}$ spectra of described compounds.

Acknowledgments: The authors gratefully acknowledge financial support for this research from the Mizutani Foundation for Glycoscience (150091) and from the National Research, Development and Innovation Office of Hungary (OTKA K 109208, K 105459 and PD 115645). The research was also supported by the EU and co-financed by the European Regional Development Fund under the project GINOP-2.3.2-15-2016-00008.

Author Contributions: M.H. and A.B. conceived and designed the experiments; E.M., D.E. and E.V. performed the experiments; E.M., M.H. and A.B. wrote the paper.

Conflicts of Interest: The authors declare no conflict of interest.

\section{References}

1. Palkin, J.S.; Eikelboom, J.W.; Cairns, J.A.; Hirsh, J. New antithrombotic agents-Insights from clinical trials. Nat. Rev. Cardiol. 2010, 7, 498-509. 
2. Gandhi, N.S.; Manecera, R.L. Heparin/Heparan sulphate-based drugs. Drug Discov. Today 2010, 15, 1058-1067. [CrossRef] [PubMed]

3. Straub, A.; Roehrig, S.; Hillisch, A. Oral, Direct Thrombin and Factor Xa Inhibitors: The Replacement for Warfarin, Leeches, and Pig Intestines? Angew. Chem. Int. Ed. 2011, 50, 4574-4590. [CrossRef] [PubMed]

4. Perzborn, E.; Roehrig, S.; Straub, A.; Kubitza, D.; Misselwitz, F. The discovery and development of rivaroxaban, an oral, direct factor Xa inhibitor. Nat. Rev. Drug Discov. 2011, 10, 61-74. [CrossRef] [PubMed]

5. Jin, L.; Abrahams, J.P.; Skinner, R.; Petitou, M.; Pike, R.N.; Carrell, R.W. The anticoagulant activation of antithrombin by heparin. Proc. Natl. Acad. Sci. USA 1997, 94, 14683-14688. [CrossRef] [PubMed]

6. Chong, B.H. Heparin-induced thrombocytopenia. J. Thromb. Haemost. 2003, 1, 1471-1478. [CrossRef] [PubMed]

7. Petitou, M.; Herbert, J.-M. A new generation of antithrombotics based on synthetic oligosaccharides. In Carbohydrate-Based Drug Discovery; Wong, C.-H., Ed.; Wiley-VCH: Weinheim, Germany, 2003; Volume 1, pp. 441-459.

8. Van Boeckel, C.A.A.; Petitou, M. A synthetic antithrombin III binding pentasaccharide is now a drug! What comes next? Angew. Chem. Int. Ed. 2004, 43, 3118-3133.

9. Westerduin, P.; van Boeckel, C.A.A.; Basten, J.E.M.; Broekhoven, M.A.; Lucas, H.; Rood, A.; van der Heiden, H.; van Amsterdam, R.G.M.; van Dinther, T.G.; Meuleman, D.G.; et al. Feasible synthesis and biological properties of six "non-glycosamino" glycan analogues of the antithrombin III binding heparin pentasaccharide. Bioorg. Med. Chem. 1994, 2, 1267-1280. [CrossRef]

10. Herczeg, M.; Lázár, L.; Borbás, A.; Lipták, A.; Antus, S. Toward synthesis of the isosteric sulfonate analogues of the AT-III binding domain of heparin. Org. Lett. 2009, 11, 2619-2622. [CrossRef] [PubMed]

11. Lázár, L.; Herczeg, M.; Fekete, A.; Borbás, A.; Lipták, A.; Antus, S. Synthesis of sulfonic acid analogues of the non-reducing end trisaccharide of the anthitrombin binding domain of heparin. Tetrahedron Lett. 2010, 51, 6711-6714. [CrossRef]

12. Herczeg, M.; Lázár, L.; Mándi, A.; Borbás, A.; Komáromi, I.; Lipták, A.; Antus, S. Synthesis of disaccharide fragments of the AT-III binding domain of heparin and their sulfonatomethyl analogues. Carbohydr. Res. 2011, 346, 1827-1836. [CrossRef] [PubMed]

13. Lázár, L.; Mező, E.; Herczeg, M.; Lipták, A.; Antus, S.; Borbás, A. Synthesis of the non-reducing end trisaccharide of the antithrombin-binding domain of heparin and its bioisosteric sulfonic acid analogues. Tetrahedron 2012, 68, 7386-7399. [CrossRef]

14. Herczeg, M.; Lázár, L.; Bereczky, Z.; Kövér, C.; Timári, I.; Kappelmayer, J.; Lipták, A.; Antus, S.; Borbás, A. Synthesis and anticoagulant activity of bioisosteric sulfonic acid analogues of the antithrombin-binding pentasaccharide domain of heparin. Chem. Eur. J. 2012, 18, 10643-10652. [CrossRef] [PubMed]

15. Herczeg, M.; Mező, E.; Lázár, L.; Fekete, A.; Kövér, K.E.; Antus, S.; Borbás, A. Novel syntheses of Idraparinux, the anticoagulant pentasaccharide with indirect selective factor Xa inhibitory activity. Tetrahedron 2013, 69, 3149-3158. [CrossRef]

16. Herczeg, M.; Mező, E.; Eszenyi, D.; Lázár, L.; Csávás, M.; Bereczki, I.; Antus, S.; Borbás, A. Synthesis of 6-sulfonatomethyl thioglycosides by nucleophilic substitution: Methods to prevent $1 \rightarrow 6$ anomeric group migration of thioglycoside 6-O-triflates. Eur. J. Org. Chem. 2013, 2013, 5570-5573. [CrossRef]

17. Mező, E.; Herczeg, M.; Eszenyi, D.; Borbás, A. Large-scale synthesis of 6-deoxy-6-sulfonatomethyl glycosides and their application for novel synthesis of a heparinoid pentasaccharide trisulfonic acid of anticoagulant activity. Carbohydr. Res. 2014, 388, 19-29. [CrossRef] [PubMed]

18. Herczeg, M.; Mező, E.; Eszenyi, D.; Antus, S.; Borbás, A. New synthesis of idraparinux, the non-glycosaminoglycan analogue of the antithrombin-binding domain of heparin. Tetrahedron 2014, 70, 2919-2927. [CrossRef]

19. Eszenyi, D.; Mándi, A.; Herczeg, M.; Bényei, A.; Komáromi, I.; Borbás, A. Synthesis of C-2- and C-3-sulfonatomethyl $O$ - and $S$-glycosides by Horner-Wadsworth-Emmons olefination. Eur. J. Org. Chem. 2016, 2016, 3884-3893. [CrossRef]

20. De Mico, A.; Margarita, R.; Parlanti, R.; Vescovi, A.; Piancatelli, G.J. A Versatile and Highly Selective Hypervalent Iodine (III)/2,2,6,6-Tetramethyl-1-piperidinyloxyl-Mediated Oxidation of Alcohols to Carbonyl Compounds. J. Org. Chem. 1997, 62, 6974-6977. [CrossRef]

21. Epp, J.B.; Widlanski, T.S. Facile Preparation of Nucleoside-5'-carboxylic Acids. J. Org. Chem. 1999, 64, 293-295. [CrossRef] [PubMed] 
22. Xia, J.; Abbas, S.A.; Locke, R.D.; Piskorz, C.F.; Alderfer, J.L.; Matta, K.L. Use of 1,2-dichloro 4,5-dicyanoquinone (DDQ) for cleavage of the 2-naphthylmethyl (NAP) group. Tetrahedron Lett. 2000, 41, 169-173. [CrossRef]

23. Wright, J.A.; Yu, J.; Spencer, J.B. Sequential removal of the benzyl-type protecting groups PMB and NAP by oxidative cleavage using CAN and DDQ. Tetrahedron Lett. 2001, 42, 4033-4036. [CrossRef]

24. Chen, C.; Yu, B. Efficient synthesis of Idraparinux, the anticoagulant pentasaccharide. Bioorg. Med. Chem. Lett. 2009, 19, 3875-3879. [CrossRef] [PubMed]

Sample Availability: For availability of samples of compounds contact the corresponding author.

(c) 2016 by the authors; licensee MDPI, Basel, Switzerland. This article is an open access article distributed under the terms and conditions of the Creative Commons Attribution (CC-BY) license (http:/ / creativecommons.org/licenses/by/4.0/). 